\title{
Abhandlung
}

\section{Wiebke Meinhold}

\section{Das Vermögen der Familie des Mannum-mēšu-lișșur}

\begin{abstract}
The family of Mannum-mēšu-lișșur belongs to the wealthy and well known families from Old Babylonian Nippur. This article will trace the development of its property through the eight documented generations spanning 150 years in total (Lipit-Enlil d to Samsu-iluna 29, ca. 1871-1721 B. C.). The study is based on 30 legal documents mentioning members of the family, and thus forming the archive of Mannum-mēšu-lișsur. Special attention will be paid to the inheritance documents.
\end{abstract}

DOI 10.1515/za-2015-0003

Die Familie des Mannum-mēšu-lișșur gehört zu den wohlhabenden Familien der altbabylonischen Stadt Nippur. ${ }^{1}$ Familienmitglieder sind in 30 Rechtsurkunden bezeugt. Mithilfe dieser Urkunden ist es möglich, die Familie und ihr Vermögen über acht Generationen und 150 Jahre hinweg zu verfolgen, in einem Zeitraum von ca. 1871 bis 1721 v. Chr. (Lipit-Enlil d bis Samsu-iluna 29). Von besonderem Interesse sind vier Erbteilungen in verschiedenen Familienzweigen und Generationen, dokumentiert durch fünf Erbteilungsurkunden. Sie bieten die Möglichkeit, das aus den älteren Generationen ererbte Vermögen mit den an die jüngeren Generationen vererbten Gütern zu vergleichen und so die Entwicklung des Familienvermögens nachzuzeichnen.

1 Dieser Artikel entstand im Rahmen des durch die DFG finanzierten Projekts „Das Erbrecht in Babylonien und Assyrien nach keilschriftlichen Quellen des 3. und 2. Jahrtausends v. Chr." Ich danke K. Volk und J. Matuszak herzlich für ihre kritische Lektüre und Diskussion des Manuskripts.

Die verwendeten Abkürzungen richten sich nach den Vorgaben des Reallexikons der Assyriologie und Vorderasiatischen Archäologie. Abweichend davon werden folgende Abkürzungen und Zeichen verwendet: PN: Personenname; PN/PN: PN, Sohn von PN; RS: Rīm-Sîn; Ha: Hammurapi; Si: Samsu-iluna; ${ }^{*}=$ kollationiert am Foto. Datumsangaben werden in der Form Regierungsjahr/Monat/Tag wiedergegeben. Absolute Jahreszahlen folgen der Mittleren Chronologie.

Wiebke Meinhold: Universität Tübingen;

Email: wiebke.meinhold@uni-tuebingen.de

\section{Forschungsgeschichte}

Die 30 Urkunden, welche Mitglieder der Familie des Mannum-mēšu-lișșur bezeugen, sind in den letzten 100 Jahren nach und nach publiziert geworden. Es handelt sich um BE 6/2, 38 und 64 (s. Poebel 1909), OECT 8, 1-2. 4-11. 16-21 (s. Hunter 1930), TIM 4, 54 (s. van Dijk 1967), MC 3, Nr. 26-28. 33. 35-37. 44. 47-48. 51 (s. Stone/Owen 1991). ${ }^{2}$

2 In MC 3, Nr. 36, einem Pfründenverkauf durch Ninurta-ēreš, Sohn von Lalûm (la-lu-ú-um), ist der Name des Käufers ist nicht erhalten. Es gibt mehrere Hinweise darauf, dass es sich um Mannum-mēšulișșur handelt: 1.) Dieser hatte entsprechende Pfründen von seinem Großvater geerbt, s. MC 3, Nr. 51: 2-6. 2.) Er kaufte entsprechende Pfründen auch von anderen Verkäufern, s. MC 3, Nr. 35, Nr. 44, OECT 8, 8. 3.) Knapp ein Jahr später kaufte er einen nahezu identischen Posten Pfründen von Warad-Ištar, Sohn von Lalûm (la-a-lum, anderer Schreiber als in MC 3, Nr. 36), wahrscheinlich einem Bruder des Ninurta-ēreš, s. MC 3, Nr. 44. 4.) Der in MC 3, Nr. 36 Rs. $4^{\prime}$ genannte Zeuge Ninurta-ēreš, Sohn von Ipqatum, verkaufte in MC 3, Nr. 35, selbst Pfründen an Mannum-mēšu-lișșur. 5.) Der in MC 3, Nr. 36 Rs. 6' erwähnte Steinschneider (bur-gul) Abbătum erscheint mehrfach in Urkunden Mannum-mēšu-lișșurs als Zeuge, s. MC 3, Nr. 35: 23, Nr. 37: 20'. Dies alles spricht dafür, MC 3, Nr. 36 zu den Urkunden des Mannum-mēšu-lișșur zu zählen.

Nicht zum Archiv des Mannum-mēšu-lișșur gehört hingegen wohl MC 3, Nr. 46, gegen Stone/Owen (1991, 25f. 82f.). Die Urkunde dokumentiert einen Pfründenkauf durch Ninurta-rā'im-zērim, den Sohn des Nannāja. Es ist keine Person genannt, welche sich sicher mit einem Mitglied der Familie des Mannum-mēšu-lișșur identifizieren ließe. Die Urkunde könnte in das Archiv des Mannum-mēšu-lișșur gelangt sein, wenn Ninurta-rā'im-zērim, der Sohn des Nannāja, ihm die Pfründe verkauft hätte. Tatsächlich verkauft ein gewisser Ninurtarā’im-zērim, Sohn von Nanna-manšum, Pfründen an Mannum-mēšulișșur, s. MC 3, Nr. 4 (Datum abgebr.) und OECT 8, 7 (Si 13/12/02). Allerdings sind diese Pfründen nicht genau identisch mit denen in 
Die Kopien und Bearbeitungen der in MC 3 publizierten Texte wurden mit geringfügigen Änderungen nochmals abgedruckt in CUSAS 15, 4. 6-8. 11-12. 16. 18-20. 23; die Kopie von MC 3, Nr. 27 aus der Hand von A. Goetze wurde nochmals abgedruckt in YOS 15, 73. Die Texte werden im Folgenden nach ihrer Erstpublikation zitiert. ${ }^{3}$

Eine ersteZusammenstellung aller ihm bekannten Texte legte Hunter (1930, 1-3) vor und unternahm den Versuch der Rekonstruktion eines Familienstammbaums. Kraus (1951, 122f.) ging kurz auf Hunters Studie ein. Eine ausführliche Untersuchung widmete der Familie Stone (1991, 19-33) mit Bearbeitung aller relevanten Urkunden. ${ }^{4}$ Sie konzentrierte sich im Wesentlichen auf den Aspekt der Adoption in der Familie des Mannum-mēšu-lișșur und berührte in diesem Zusammenhang auch Fragen nach dem Familienvermögen.

Die folgende Untersuchung macht neue Vorschläge zur Rekonstruktion des Familienstammbaums. Sie lassen die Entwicklung des Familienvermögens in einem neuen Licht erscheinen.

\section{Das Archiv des Mannum-mēšu- lișșur}

Der Begriff „Archiv“ bezeichnet Alltagsdokumente, die zusammen aufbewahrt wurden und sich im Besitz einer Person, Familie oder Institution befanden. ${ }^{5}$ Es stellt sich die Frage, ob die hier zur Diskussion stehenden 30 Urkunden in diesem Sinne ein Archiv bildeten und wem sie gehörten. ${ }^{6}$

Für die Beurteilung von Archivzusammenhängen ist es wichtig zu wissen, dass Urkunden, die einen Erwerbsvorgang dokumentierten - sei es Kauf, Tausch, Erbe oder Schenkung -, in altbabylonischer Zeit und darüber hinaus stets für den Erwerbenden bestimmt waren, damit

MC 3, Nr. 46, und es ist fraglich, ob Ninurta-rā'im-zērim, Sohn von Nannāja, und Ninurta-rā'im-zērim, Sohn von Nanna-manšum, identisch sind. Somit gibt es keinen sicheren Anhaltspunkt für eine $\mathrm{Zu}$ gehörigkeit von MC 3, Nr. 46 zum Archiv des Mannum-mēšu-lișșur.

3 Die Bearbeitungen, Kopien und Fotografien in Stone/Owen (1991) findet man über die ebd., 93, gegebene Konkordanz.

4 Für Korrekturen zu diesen Textbearbeitungen s. die Rezensionen von Van De Mieroop (1991-1993) und Charpin (1994). Vgl. auch die Rezensionen von Oelsner (1993), Obermark (1993/1994) und Otto (1995). Die Korrekturen der Rezensenten wurden leider bei dem erneuten Abdruck der Texte in CUSAS 15 kaum berücksichtigt.

5 So mit Pedersén (1998, 2f.).

6 Kraus $(1951,122)$ meinte, 11 von 18 ihm bekannten Urkunden hätten Mannum-mēšu-lișșur selbst, 7 seinen Verwandten gehört. Die gesamte Textgruppe bezeichnete er als „Archiv der Familie des Mannum-mêšu-lișșur“. Stone/Owen (1991, Titel und passim) hingegen sprachen vom „archive of Mannum-mešu-lișșur“. dieser seine Rechte an der erworbenen Sache nachweisen konnte. Der Erwerber erhielt zugleich vom Veräußerer auch alle älteren Urkunden, die sich auf frühere Veräußerungen der fraglichen Sache bezogen. Ältere Erwerbsurkunden begleiteten also den Erwerbsgegenstand und wechselten gemeinsam mit diesem den Besitzer. ${ }^{7}$

Dies vorausgesetzt, waren offensichtlich alle Urkunden im Besitz des Mannum-mēšu-lișșur, welche seine Erwerbungen dokumentierten, nämlich 18 Kaufurkunden, in denen Mannum-mēšu-lișșur als Käufer fungierte, und eine Tauschurkunde, in der er als Tauschpartner auftrat. ${ }^{8}$ Ebenfalls im Besitz Mannum-mēšu-lișșurs waren wohl drei weitere Urkunden: ein Ehevertrag mit Adoption seitens Nannāja, bei der Mannum-mēšu-lișșur eine der adoptierten und als Erben eingesetzten Personen war, eine Erbteilungsurkunde bezüglich des Nachlasses von Nuska-amah, durch die er anstelle seines verstorbenen Vaters Awilija dessen Erbteil erhielt, sowie eine Prozessurkunde, in welcher er Ausgaben für Ibbi-Enlil erfolgreich von dessen Söhnen zurückforderte. ${ }^{9}$

Fünf Urkunden, die ursprünglich für Vorfahren und Verwandte Mannum-mēšu-lișșurs ausgestellt worden waren, gelangten wahrscheinlich zusammen mit den darin verzeichneten Gütern in den Besitz Mannum-mēšulișșurs. Dazu zählt eine Urkunde (OECT 8, 20), welche die Adoption von Mannum-mēšu-lișșurs Ururgroßvater Lu-Bau dokumentiert, ca. 130 Jahre vor der frühesten Bezeugung Mannum-mēšu-lișșurs. Sie ist vermutlich ebenso wie ein Exemplar der Erbteilungsurkunde bezüglich des Nachlasses von Lu-Bau ${ }^{10}($ OECT 8,17$)$ zusammen mit dem vererbten Familienbesitz durch die Generationen weitergegeben worden und schließlich auf Mannum-mēšulișșur gekommen. Das andere Exemplar der Erbteilungsurkunde (OECT 8,18$)$ gehörte ursprünglich vielleicht dem Familienzweig von Lu-Baus jüngerem Sohn Adda-kalla. ${ }^{11}$

7 Siehe dazu Charpin (1980, 156-159; 1986, 121-140); Janssen/ Gasche/Tanret (1994, 96-110).

8 Kauf: BE 6/2, 38; OECT 8, 1, 2, 5, 6, 7, 8, 9, 10; TIM 4, 54; MC 3, Nr. 44, 35, 47, 33, 48, 28, 36 (Name des Käufers hier nicht erhalten, sehr wahrscheinlich Mannum-mēšu-lișșur s. Anm. 2), 37; Tausch: OECT 8, 16.

9 Ehevertrag mit Adoption durch Nannāja: MC 3, Nr.27. Erbteilung Nachlass Nuska-amah̆: MC 3, Nr. 51. Prozessurkunde: OECT 8, 11.

10 Für Lu-Bau als Erblasser, nicht Ur-Lumma, s. Abschnitt 3.1.

11 OECT 8, 18: 41-43 enthält einen Passus über eine Ausgleichszahlung, welche Adda-kalla an Nanna-meša zahlte. Das lässt vermuten, dass OECT 8, 18 für Adda-kalla ausgefertigt worden war, OECT 8, 17 ohne diesen Passus hingegen für Nanna-meša. Allerdings gibt es noch kleinere Unterschiede in der Auflistung der ererbten Pfründen und bei der Siegelung der Urkunden. Deshalb ist auch nicht ganz auszuschließen, dass die eine Urkunde eine Korrektur der anderen darstellen sollte - so Hunter (1930, 29); Klíma (1940, 24) - und beide Urkunden für einen der zwei Erben bestimmt waren. 
Mannum-mēšu-lișșur könnte das Exemplar erhalten haben, weil er große Teile des Besitzes dieses Familienzweiges von den Nachfahren des Adda-kalla erwarb. ${ }^{12}$ Aus diesem Grund gelangten vermutlich auch die Erbteilungsurkunden bezüglich der Nachlässe von Adda-kallas mutmaßlichem Sohn bzw. Enkelsohn, Sîn-iqišam und IbbiEnlil, in seinen Besitz (MC 3, Nr. 26; OECT 8, 19). ${ }^{13}$

Bei zwei Urkunden ist es unsicher, ob sie je Mannummēšu-lișșur gehörten, wenngleich entsprechende Szenarien denkbar sind. Die ältere der beiden beurkundet einen Klageverzicht von Awilija und Munawwirum bezüglich einer Haus-Schenkung ihres Großvaters Nanna-meša an ihre Tante Nin-kuzu, für die ihr Vater Nuska-amah eine Abfindung erhalten hatte (OECT 8, 4). ${ }^{14}$ Die Urkunde dokumentiert noch eine weitere Ausgleichszahlung, welche nahelegt, dass die Tafel ursprünglich für Aham-arši, vermutlich einen Cousin von Awilīja und Munawwirum, ausgestellt worden war: Aham-arši leistete diese zweite Ausgleichszahlung dafür, dass er 10 Tage im Jahr des Pfründenamtes (nam-gudu $_{4}$ den-líl $\mathrm{u}_{18}$-ru-mahan-ki behalten konnte. Pfründen galten in altbabylonischer Zeit als wertvoller Besitz. Sie garantierten ihren Inhabern ein regelmäßiges Einkommen für die Erfüllung von Aufgaben im Rahmen des Tempelbetriebs. Sollte Mannum-mēšu-lișșur die entsprechende Pfründe später von Aham-arši erworben haben, wäre auch die Urkunde in seinen Besitz gekommen. Tatsächlich kaufte Mannummēšu-lișșur ein Wohnhausgrundstück von der Tochter und der Frau eines offenbar ohne männliche Erben verstorbenen Aham-aršis (OECT 8, 1), der mit Aham-arši, seinem Onkel zweiten Grades identisch sein könnte. Es besteht die Möglichkeit, dass Mannum-mēšu-lișșur auf diesem Wege auch die Pfründen Aham-aršis erwarb und so die fragliche Urkunde in seinen Besitz kam.

Die andere Urkunde, deren Zugehörigkeit zum Archiv des Mannum-mēšu-lișșur nicht sicher festzustellen ist,

12 Siehe MC 3, Nr. 27 (Si 6/07/-; Nannāja heiratet Lamassum und adoptiert vier Männer, vermutlich ihre Kinder. Er vermacht seinen Adoptivsöhnen, darunter Mannum-mēšu-lișșur, sein ererbtes Vermögen, s. Anm. 35.), MC 3, Nr. 28 (Si 08/01/14; Mannum-mēšu-lișșur kauft Ibbi-Enlil/Sîn-iqī̌sam seine ererbten $44 \mathrm{~g}$ i $\hat{\mathrm{g}}_{4}$ Wohnhaus ab.), OECT 8, 11 (Si 10/09/03; Mannum-mēšu-lișșur erhält infolge eines Prozesses als Entschädigung für Getreide- und Nahrungslieferungen an Ibbi-Enlil von dessen drei Söhnen einen Monat der Pfründe é-da-di den-líl dnin-1íl.), MC 3, Nr. 33 (Si 11/02/01; Mannummēšu-lișșur kauft Ninurta-muštāl/Ibbi-Enlil seine ererbten Pfründen ab.), MC 3, Nr. 37 (Si 11/12/-; Mannum-mēšu-lișșur kauft é-da-di Pfründe von Inbi-ilišs/Ninurta-muštāl und dessen Mutter Lamassatum. Lies Rs. $14^{\prime}$ : é-d a ${ }^{*}-d i^{*}$-bi-šè enim nu-um-ĝá-ĝá-a).

13 Zur Lesung des Namens Ibbi-Enlil s. Anm. 55.

14 Für eine Diskussion dieses Textes s. Abschnitt 3.3. dokumentiert die Adoption von Mannum-mēšu-lișșurs Bruder Ninurta-muballiț. durch ein Ehepaar (OECT 8, 21). ${ }^{15}$ Die Urkunde war zunächst wohl im Besitz des adoptierten Bruders. Sie könnte in die Hände Mannum-mēšu-lișșurs gelangt sein, wenn dieser das seinem Bruder seitens der Adoptiveltern verschriebene Erbe in irgendeiner Weise erworben hätte, wofür es allerdings keine Belege gibt.

Einzig eine Urkunde mit Erwähnung Mannum-mēšulișșurs war mit Sicherheit nie in seinem Besitz: BE 6/2, 64 regelt den Rückkauf eines unbebauten Hausgrundstücks (é kislaḩ) nach dem Tod des Mannum-mēšu-lișșur, denn als Verkäufer treten sein Bruder, sein Sohn und seine Ehefrau auf. Die Urkunde wurde für den Käufer Ninurtarā'im-zērim, Sohn von Ninurta-manšum, ausgestellt und ging zusammen mit dem Grundstück in dessen Besitz über.

Somit ergibt sich folgendes Bild: 22 Urkunden waren speziell für Mannum-mēšu-lișșur ausgestellt worden. Fünf Urkunden, welche ursprünglich seinen Vorfahren und Verwandten gehörten, gelangten wahrscheinlich gemeinsam mit den darin dokumentierten Gütern durch Erbe oder Kauf in den Besitz von Mannum-mēšu-lișșur. Bei zwei Urkunden ist dies unsicher, wenngleich nicht unmöglich. Nur für eine Urkunde lässt sich zeigen, dass sie nicht im Besitz des Mannum-mēšu-lișșur war. Zumindest 27, vielleicht sogar 29 der insgesamt 30 Urkunden können also mit gutem Recht als „Archiv des Mannum-mēšulișșur" bezeichnet werden: Sie waren in seinem Besitz und dienten ihm als Nachweis seiner Eigentumsrechte an den darin dokumentierten Gütern.

Dieser Umstand ist bei dem Versuch, Geschichte und Entwicklung des Vermögens der Familie Mannum-mēšulișșurs über acht Generationen hinweg nachzuzeichnen, $\mathrm{zu}$ berücksichtigen. ${ }^{16}$ Man muss damit rechnen, dass wichtige Dokumente und Bestandteile des Familienvermögens nach wie vor unbekannt sind.

Wo das Archiv des Mannum-mēšu-lișșur in Nippur zutage kam, ist leider nicht bekannt: Die Urkunden, die heute in Oxford, Bagdad und Ithaca aufbewahrt werden, sind über den Kunsthandel in die jeweiligen Sammlungen gelangt. Die beiden in Philadelphia befindlichen Urkunden BE 6/2, 38 und 64 wurden 1889-1890 im Verlauf der ersten beiden Grabungskampagnen der University of Pennsylvania in Nippur ausgegraben und stammen wohl vom „Tablet Hill“ im Südosten der Stadt. Die Angaben zu ihrem Fundort sind aber leider sehr vage. ${ }^{17}$

$15 \mathrm{Zu}$ dieser Urkunde s. Abschnitt 3.4 mit Anm. 41-42.

16 Auf die Gefahren, die damit verbunden sind, älteres Familienvermögen auf Grundlage eines jüngeren Archivs rekonstruieren zu wollen, hat u. a. Charpin $(1986,139)$ hingewiesen.

17 Poebel $(1909,1)$ notierte zum Fundort der in BE 6/2 publizierten 


\section{Stammbaum und Familiengeschichte}

Ziel des folgenden Abschnitts ist die Rekonstruktion eines verlässlichen Stammbaums (s. Anhang 2) mit Diskussion aller Unsicherheiten. Für ältere Rekonstruktionsversuche s. Hunter $(1930,1)$ und Stone/Owen $(1991,20)$.

\subsection{Generationen 1-4: Die Adoption des Lu-Bau und die Teilung seines Nachlasses}

Das älteste bekannte Familienmitglied ist Lu-Inana (Generation 1). Er ist allerdings nur in der Filiationsangabe seines Sohnes Ur-Lumma bezeugt. Ur-Lumma ist das älteste bekannte, aktive Familienmitglied (Generation 2). Er adoptierte im Jahr Lipit-Enlil d (ca. 1875-1871) einen Mann namens Lu-Bau (Generation 3), s. OECT 8, 20. Die zeitlich nächstfolgenden Dokumente sind die weitgehend parallelen Urkunden OECT 8, 17 und OECT 8, 18 (Sîniqišam 3 oder 4/12/-, ca. 1838/1837). Ihnen zufolge teilten ca. 35 Jahre nach der Adoption des Lu-Bau die Brüder Nanna-meša und Adda-kalla, die Erben des Lu-Bau, einen Nachlass zu gleichen Teilen (Generation 4).

Es stellt sich die Frage, wer der Erblasser dieses Nachlasses war. In der Urkunde ist er als solcher nicht ausdrücklich benannt. Mitchell $(1991,96)$ und Van De Mieroop (1991-1993, 127b) gingen davon aus, der Erblasser sei Ur-Lumma gewesen. Das ist aus drei Überlegungen heraus unwahrscheinlich: 1.) Ur-Lumma adoptierte den Lu-Bau bereits ca. 35 Jahre zuvor als erwachsenen Mann für seine eigene Altersversorgung. Der Zeitraum von 35 Jahren erscheint recht lang für einen Lebensabend, in welchem der Adoptivvater Ur-Lumma auf Versorgung durch seinen Adoptivsohn Lu-Bau angewiesen gewesen sein sollte. 2.) Eine Position des geteilten Nachlasses ist ein Garten aus dem Erbteil des Ur-Lumma. ${ }^{18}$ Das bedeutet aber keineswegs, dass es sich bei allen genannten Gütern um den Nachlass des Ur-Lumma handeln muss. Wäre dies der Fall, wäre die Spezifizierung des Gartens als Erbteil

Tontafeln: „As Prof. Hilprecht informs me, they were found for the greater part in the southwest section of Mound IV (cf. the map of the ruins of Nippur in Hilprecht, B. E., Series D, Vol. I, p. 305) during the first and second expeditions of the University of Pennsylvania." Auf der von Poebel zitierten Karte ist der „Tablet Hill“ mit der Zahl IV markiert.

18 OECT 8, 17: 19f. 41f. // OECT 8, 18: 16f. 38f. (s. Stone/Owen 1991, Nr. 24 // Nr. 53): šà ĝšškiri ${ }_{6}$ PI-ru-ru-tum ḩa-la-ba ur-dlum-ma šu-ri-a-bi in-ba-e-eš, „Das Innere eines ... Gartens, Erbteil des Ur-Lumma, haben sie jeweils zur Hälfte geteilt.“ des Ur-Lumma wohl überflüssig. 3.) Beide Exemplare der Erbteilungsurkunde sind gesiegelt durch die Erben: OECT 8, 17 mit einem gemeinsamen Siegel: ${ }^{1}$ dn a n na-me-ša ${ }_{4}$ ${ }^{2}$ ad-da-kal-la ${ }^{3} i b i l a ~ l u ́-d b a-u ́,$, Nanna-meša (und) Adda-kalla, die Erben von Lu-Bau“; OECT 8, 18 mit zwei Siegeln: ${ }^{1}$ dnanna-me-ša ${ }_{4}{ }^{2} d u m u$ lú-dba-ú bzw. 1ad-da-kal-la ${ }^{2}$ dumu lú-dba-ú, „Nanna-meša, Sohn von Lu-Bau“ bzw. „Adda-kalla, Sohn von Lu-Bau“. Da die Erben in den Siegelinschriften ausdrücklich als Erben und Söhne von Lu-Bau bezeichnet werden, ist es höchstwahrscheinlich dessen Nachlass - der Nachlass ihres Vaters Lu-Bau -, den sie teilten.

Stone (1991, 21 mit Anm. 39) ging davon aus, dass der Erblasser Lu-Bau war, nahm aber an, Adda-kalla und Nanna-meša seien Söhne von Ur-Lumma und Adoptivbrüder des von Ur-Lumma adoptierten Lu-Bau. ${ }^{19}$ Sie bot dafür zwei Argumente: 1.) Die Erbteilungsurkunden OECT 8, 17 // 18 verzeichnen keinen Vorzugsanteil. Das wertete Stone als Indiz für eine Unregelmäßigkeit im Verhältnis zwischen Erblasser und Erben dahingehend, dass die Erben Adoptivbrüder des Erblassers seien. 2.) Die Erben Adda-kalla und Nanna-meša sind angeblich im Urkundentext und im Siegel nur als i bila von Lu-Bau bezeichnet, nicht als du mu.

Warum Nanna-meša als älterer Bruder keinen Vorzugsanteil erhielt, ist unklar. Anders als von Stone behauptet, ist dies nicht der einzige Beleg für eine Erbteilung ohne Vorzugsanteil aus Nippur. ${ }^{20}$ Die Gründe für diesen Teilungsmodus sind noch zu untersuchen. Es ist nicht auszuschließen, dass Adoption eine Rolle spielte. Dass aber jede derartige Erbteilung den Nachlass eines (Adoptiv)bruders zum Gegenstand hatte, ist zu bezweifeln, da es bislang überhaupt keinen sicheren altbabylonischen Beleg für die Teilung eines brüderlichen Nachlasses gibt. ${ }^{21}$ Stone's zweites Argument, Adda-kalla und Nanna-meša seien nur als ibila von Lu-Bau bezeichnet, trifft nicht zu. Aus den gerade zitierten Siegelinschriften ist ersichtlich, dass Nanna-meša und Adda-kalla in OECT

19 Ähnlich schon Hunter (1930, 1. 29), der allerdings in Lu-Bau den (Adoptiv-)Vater sah und in Ur-Lumma den Erblasser und Adoptivbruder der Erben Nanna-meša und Adda-kalla.

20 Vgl. außerdem BE 6/2, 44 (Si 14/11/-, Erbteilung unter zwei Söhnen des Erblassers) und BE 6/2, 28 (Si 08/03/-; Erbregelung im Rahmen einer Adoption); evtl. auch ARN 33 + PBS 8/1, 29 (RS 35/3?/-, Aufteilung eines Nachlasses auf zwei Gruppen zu je zwei Erben), ARN 144 + OIMA 1, 54 ([...], nach Si 13/02/01, Teilung eines Nachlasses unter evtl. dem Bruder? des Erblassers und den Söhnen des Adoptivsohnes des Erblassers), ARN 143 ([[...], Erbteilung unter vier Erben, fragmentarisch), ARN 49 (RS 30+x, fragmentarisch).

21 Klíma (1940, 24) führte als Beleg einer Erbteilung nach dem Bruder nur OECT 8, 17 // 18 an; in der Interpretation folgte er Hunter (1930, 1. 29). 
8, 17 als ibila, „Erben“, in OECT 8, 18 aber jeweils als d u m u, „Sohn“, von Lu-Bau bezeichnet sind. Die Begriffe ibila und dumu sind hier offensichtlich synonym zur Bezeichnung erbberechtigter Söhne gebraucht. ${ }^{22}$

Dass Nanna-meša und Adda-kalla die Söhne von Lu-Bau waren, nicht seine Adoptivbrüder, lässt sich noch durch eine weitere Überlegung erhärten: Ur-Lumma adoptierte Lu-Bau gegen Stone $(1991,21)$ nicht aus wirtschaftlicher Not oder wegen Unveräußerbarkeit der Pfründen in seinem Besitz, denn das Vermögen, welches er ihm in der Adoptionsurkunde OECT 8, 20 vermachte, war umfangreich. Es bestand aus Pfründen, aber auch aus - mengenmäßig unspezifiziert - Haus, Feld und sonstiger Habe, soviel vorhanden. Im Gegenzug leistete der Adoptivsohn Lu-Bau nicht etwa eine Zahlung oder Schuldenbegleichung, wie es bei einer Adoption aus wirtschaftlicher Not heraus zu erwarten wäre, ${ }^{23}$ sondern er verpflichtete sich zu regelmäßigen Unterhaltszahlungen an seinen Adoptivvater Ur-Lumma. Klageverzicht leisteten „Ur-Lumma und seine Erben, so viele es geben mag“" ${ }^{24}$ Der Umstand, dass es sich hier um eine Adoption mit Vermögensübertragung gegen Unterhaltszahlungen handelt und dass im Klageverzicht keine Erben namentlich genannt sind, legt die Vermutung nahe, dass der adoptierende Ur-Lumma keine Söhne und auch sonst keine Erben hatte. Wären Nannameša und Adda-kalla seine Söhne gewesen, warum hätte er jemandem außerhalb seiner Familie sein ansehnliches Vermögen vermachen sollen, um Unterhalt im Alter zu erhalten? Es ist vielmehr davon auszugehen, dass UrLumma den Lu-Bau adoptierte, weil er einen Erben benötigte, der seine Altersversorgung übernahm. Nanna-meša und Adda-kalla hingegen waren die Söhne von Lu-Bau, dessen Nachlass sie nach seinem Tod teilten.

\subsection{Generationen 4-8 im Familienzweig des Adda-kalla: Die Nachkommen Adda-kallas}

Nachkommen Adda-kallas sind mit entsprechender Filiation nicht bezeugt. Einen Hinweis auf seine Söhne bietet aber möglicherweise die Erbteilungsurkunde MC 3, Nr. 26. Sie dokumentiert eine Teilung des Nachlasses von Sîniqī̌am und zusätzlich auch des ererbten Besitzes seines

22 Siehe dazu auch Obermark (1992, Part I, 58-65).

23 Vgl. z.B. den Adoptionsvertrag BE 6/2, 28, gemäß dem der Adoptivsohn am Tage der Adoption eine Schuld von 4 Kor Gerste für seinen Adoptivvater beglich.

24 OECT 8, 20 Rs. 21f. (s. Stone/Owen 1991, Nr. 23): ur-dlu m-ma ù ibila-a-ni a-na me-a-bi.
Bruders Ilī-āpilī. Der gesamte geteilte Besitz entspricht hinsichtlich Art und Menge in etwa dem Erbteil von Addakalla, s. Abschnitt 4.2. Das legt die Vermutung nahe, dass Sîn-iqiišam und Ilī-āpilī die Söhne Adda-kallas waren und die Generation 5 in dessen Familienzweig bildeten. ${ }^{25}$

Generation 6 ist vertreten durch Sîn-iqiı̌šms Söhne, Ibbi-Enlil und Nannāja. Ibbi-Enlil, seine Söhne und ein Enkelsohn verkauften Vermögen an Mannum-mēšulișșur. Nannāja schuf durch Heirat und Adoption eine Verbindung mit dem Familienzweig des Nanna-meša, s. Abschnitt 3.4 .

\subsection{Generationen 4-6 im Familienzweig des Nanna-meša: Klageverzicht von Awìlija und Munawwirum bezüglich einer Schenkung ihres Großvaters Nanna-meša}

Ca. 75 Jahre nach der Teilung des Nachlasses von Lu-Bau unter Nanna-meša und Adda-kalla datiert OECT 8, 4 (Ha 31/05/-, ca. 1762). Die Urkunde dokumentiert den Klageverzicht einiger Erben des Nanna-meša in zwei Angelegenheiten. Hintergrund der ersten Angelegenheit war offenbar folgender: Nanna-meša (Generation 4) hatte seiner Tochter Nin-kuzu (Generation 5) zu Lebzeiten 15 $\mathrm{gi} \hat{\mathrm{g}}_{4}\left(9 \mathrm{~m}^{2}\right)$ Wohnhaus geschenkt. Nach dem Tod von Nanna-meša klagten Awilija und Munawwirum (Generation 6), die Söhne Nuska-amahss, Enkel Nanna-mešas und Neffen der beschenkten Nin-kuzu, weil sie sich durch diese Schenkung offenbar in ihrer Erbschaft benachteiligt sahen. Anlass zu dieser Klage gaben wahrscheinlich der Tod ihres Vaters Nuska-amah und der Antritt seines Erbes. Doch hatte Nuska-amah zu seinen Lebzeiten eine Ausgleichszahlung in Höhe von 5 Schekeln Silber für die Schenkung seines Vaters Nanna-meša an seine Schwester Nin-kuzu erhalten - möglicherweise im Zusammenhang mit der Teilung des Nachlasses von Nanna-meša. Die Ausgleichszahlung wurde von vier Personen gezahlt: Ahamarši, Sohn von Sîjjatum, Ipqu-erșetim, Sohn von Tillatī, Ina-šamê-wussum und Nuska-amah, beide ohne Filiation. Wegen dieser bereits geleisteten Ausgleichszahlung an ihren Vater bekamen die Kläger kein Recht und mussten einen zukünftigen Klageverzicht beeiden.

25 So auch Stone/Owen (1991, 20. 29). Gegen Stone/Owen ist $i$-li- $a$ PI-li nicht „Ili-awili“ zu lesen, sondern Ilī-āpilī, „Mein Gott ist einer, der mich befriedigt.“, s. AHw 56b, apālu(m) I 1g. Ilī-āpilī ist als Zeuge auch noch einmal in MC 3, Nr. 27 Rs. 11' bezeugt. Dort sind Spuren vom ersten Zeichen seines Vaternamens erhalten, die durchaus für 'AD' sprechen könnten, s. Foto Stone/Owen (1991) Pl. 2. 
Tabelle 1: Erbteilung von Wagen in TIM 4, 1

\begin{tabular}{llll}
\hline Erbe 1 & Erbe 2 & Erbe 3 & Erbe 4 \\
\hline unvollendeter Wagen & alter Wagen & neuer Wagen & (kein Wagen) \\
$+1,5$ Schekel Silber & -1 Schekel Silber & -4 Schekel Silber & +1 Schekel Silber von Erbe 2 \\
šà m l & an Erben 4 & an Erben 4 & \\
& & & +4 Schekel Silber von Erbe 3 \\
\hline
\end{tabular}

Die zweite in der Urkunde dokumentierte Rechtsangelegenheit besteht in der Leistung einer Ausgleichszahlung für 10 Tage der Pfründe den-líl $\mathrm{u}_{18}$-ru-mah-an-ki seitens Aham-arši an Ipqu-erșetim, Awilija und Munawwirum. Die drei Empfänger beeiden einen Klageverzicht bezüglich dieses Silbers.

Stone (1991, 21f.) meinte, die beiden erfolglosen Kläger im ersten Rechtsakt, Awilija und Munawwirum, seien Adoptivsöhne des Nuska-amah, nicht leibliche Söhne, weil sie sowohl im Urkundentext als auch in der Siegelinschrift als ibila des Nuska-amah bezeichnet seien, nicht als d u m u. Das trifft nicht zu. Zum einen sind Awilija und Munawwirum gegen Stone in der Siegelinschrift als du mu bezeichnet. ${ }^{26}$ Zum anderen bilden die Begriffe ibila und dumu keinen Anhaltspunkt für die Art des Kindschaftsverhältnisses: sie werden synonym verwendet sowohl für leibliche als auch für Adoptivkin$\operatorname{der}^{27}$. Zum Nachweis eines Adoptionsverhältnis gibt es nur zwei sichere Möglichkeiten: entweder eine Adoptionsurkunde oder zwei verschiedene Vatersnamen bei nachgewiesenermaßen demselben Individuum. Solange keiner dieser Nachweise für Awilìja und Munawwirum erbracht werden kann, wird von einer leiblichen Sohnschaft zu Nuska-amah ausgegangen.

Stone ging nicht der Frage nach, wer die vier Personen waren, welche die Ausgleichszahlung im ersten Abschnitt der Urkunde leisteten. Zur Beantwortung dieser Frage ist ein Exkurs zur Bezeichnung der Ausgleichszahlung als šà mul é ad-da-na notwendig. ${ }^{28}$ Dieser Ausdruck ist noch in zwei weiteren Erbteilungsurkunden bezeugt: in ARN 46 für eine Ausgleichszahlung bestehend aus Feld,

26 Lies gemäß dem Foto vom linken Rand, Stone/Owen 1991, Pl. 32:

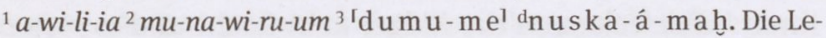
sung ' $\mathrm{d} u \mathrm{mu}-\mathrm{me}^{\mathrm{I}}$ ist trotz unvollständigem Abdruck des Siegels in dieser Zeile die einzig mögliche, denn für i bila, d. h. für die Zeichen DUMU-NITA, reicht der Platz nicht aus.

27 Obermark (1992, Part I, 58-65, z.B. 61): „Indeed, the terms DUMU:māru and IBILA:aplu seem to function interchangeably in the contracts. Our conclusion here is that in the context of adoption, at least, there was no practical distinction between mārūtum and aplūtum."

28 OECT 8, 4: 5 (s. Stone/Owen 1991, Nr. 25). unbebautem Hausgrundstück und einem Sklaven im Gesamtwert von einer Mine Silber und in TIM 4, 1 für eine Ausgleichszahlung für die Verteilung von Wagen verschiedener Qualität unter vier Erben (s. Tabelle 1): Der älteste Erbe erhielt einen unvollendeten Wagen, der zweite Erbe einen alten, der dritte einen neuen, der vierte keinen Wagen. Dafür bekam der vierte Erbe insgesamt 5 Schekel Silber an Ausgleichszahlungen vom dritten und zweiten Erben. Der erste Erbe erhielt zusätzlich zu seinem unvollendeten Wagen 1,5 Schekel Silber, die als šà mul bezeichnet sind.

Die Ausgleichszahlungen für den vierten Erben stammen von seinen Miterben. Entsprechend könnte šà mul die Herkunft der Ausgleichszahlung für den ersten Erben bezeichnen, genauer das Vermögen, dem diese entnommen wurde. Vielleicht handelt es sich dabei um den gesamten Nachlass vor der Teilung.

Der Ausdruck šà mul findet sich auch in der lexikalischen Serie Ana ittišu (Landsberger 1937, 39, Tf. 3 ii 29-31):

\begin{tabular}{|c|c|c|}
\hline šà mul & lìb-bi ši-iț-ri & „Herz/Inhalt der Schrift“ \\
\hline šà mul é ad-da & II É A.BA & $\begin{array}{l}\text { „Herz/Inhalt der Schrift“ } \\
\text { des Vatershauses }\end{array}$ \\
\hline mul é & III É A.BA & $\begin{array}{l}\text { „Herz/Inhalt der Schrift“ } \\
\text { des Vatershauses }\end{array}$ \\
\hline šu & il-qe & hat er erhalten \\
\hline
\end{tabular}

Landsberger $(1937,145)$ vermutete, šà $\mathrm{mul}=$ libbi šiți sei „ein Dokument über die künftige oder schon vorgenommene Erbteilung, also ein Testament oder eine Erbteilungsurkunde“. Die Deutung „Testament“ übernahm Prang $(1976,19)$. Die Wörterbücher äußerten sich vorsichtiger: Laut AHw. 550a, libbu $(m)$ B 1, ist die Bedeutung von libbi šițri unklar; CAD L, 169, libbu $2 \mathrm{~g}$, verallgemeinerte Landsbergers Annahme mit der Angabe „a type of document". ${ }^{29}$

Eine Bedeutung „Testament“ erscheint eher unwahrscheinlich: allgemein, weil aus altbabylonischer Zeit

29 Vgl. CAD Š III, 145a, šițru lexical section: „written document“ bzw. „written document concerning the paternal estate“. 
bislang kein einziges Testament überliefert ist; und speziell, weil in TIM 4, 1 ein beträchtliches Vermögen vererbt wird (außer den Wagen noch Wohnhaus, unbebautes Hausgrundstück, Feld, Vieh, Pfründe, Mahlsteine, Türen). šà mul nimmt jedoch nur Bezug auf 1,5 Schekel Silber als Ausgleichszahlung für einen unvollständigen Wagen. Warum sollte es ein Testament für diesen verhältnismäßig geringen Betrag gegeben haben, nicht aber für das erheblich größere sonst vererbte Vermögen?

Die vorgeschlagene Deutung von šà $\mathrm{mul}$ als einer Art von Vermögen erfährt Unterstützung durch den Kontext in Ana ittišu. Der entsprechende Abschnitt (Landsberger 1937, 38-40, Tf. 3 ii 17-40) behandelt in erster Linie verschiedene Arten von Vermögen im Zusammenhang mit unterschiedlichen Rechtsgeschäften (z. B. kù ka-kéš- da: kasap kiṣri, „Silber (aus) der Miete“, kù u $_{5}$-t uku: kasap hubulli, „Silber (aus) einer verzinslichen Schuld“), Vermögenszuwachs und -verlust. Sollte šà mul, akkadisch libbi šitri, wörtlich „Herz/Inhalt der Schrift“, tatsächlich ein Dokument bezeichnen, dann vielleicht ein NachlassInventar, welches das gesamte vererbte Vermögen vor der Teilung enthielt. ${ }^{30}$

Trifft diese Deutung zu, so entstammte die in der oben diskutierten Klageverzichturkunde (OECT 8, 4) erwähnte Ausgleichszahlung von 5 Schekeln Silber an Nuska-amah dem noch ungeteilten Nachlass seines Vaters Nannameša, vielleicht gemäß einem nicht erhaltenen Inventar dieses Nachlasses. Tätigen konnten eine solche Ausgleichszahlung wohl nur alle Erben des Nanna-meša gemeinsam. Das würde bedeuten, dass Aham-arši, der Sohn von Sîjjatum, Ipqu-erșetim, der Sohn von Tillatī, Inašamê-wussum und Nuska-amah, beide ohne Filiation, die Erben von Nanna-meša waren - Ina-šamê-wussum und Nuska-amah vermutlich seine Söhne, Aham-arši und Ipqu-erșetim hingegen Enkelsöhne, die den Erbteil ihrer vermutlich zum Zeitpunkt der Erbteilung schon verstorbenen Väter erhielten. Alle Erben gemeinsam, unter ihnen auch Nuska-amah selbst, hätten demnach aus dem Nachlass ihres Vaters bzw. Großvaters vor der Erbteilung für Nuska-amah - und möglicherweise nicht nur für ihn, sondern für einen jeden von ihnen - eine Ausgleichszahlung wegen der früheren Schenkung des Erblassers an seine Tochter entnommen. Anlass zu dieser Ausgleichszahlung gab vermutlich die anstehende Teilung des Nachlasses von Nanna-meša. Dieser Rekonstruktion zufolge war Nuska-amah nicht der einzige Sohn von Nanna-meša,

30 Klare Belege für derartige Inventare sind bislang nicht bekannt. Verwiesen werden kann aber auf Entwürfe für Erbteilungsurkunden, S. z. B. Lafont (1992, 103-105. 110); Kraus (1951, 185. 199-203), Texte C (ARN 58, Ni 2182) und D (Ni 2181). sondern hatte drei Brüder, Sîjjatum, Tillatī und Ina-šamêwussum, sowie eine Schwester, Nin-kuzu.

Betrachten wir noch kurz die zweite auf der Urkunde dokumentierte Rechtsangelegenheit: Aham-arši leistete eine Ausgleichszahlung an Ipqu-erșetim, Awilija und $\mathrm{Mu}-$ nawwirum für eine Pfründe. Hintergrund könnte auch hier die vorangegangene Teilung des Nachlasses von Nannameša sein. Doch für wen war die Urkunde ausgestellt? Im zweiten Fall offenbar für Aham-arši als Beleg für seine Ausgleichszahlung und den Klageverzicht seiner Miterben. Entsprechend ist anzunehmen, dass die Urkunde auch im ersten Fall für Aham-arši ausgestellt war, der vielleicht Sohn des ältesten Erben war und als solcher mit der Verwaltung des gesamten Nachlasses und den Teilungsmodalitäten beauftragt gewesen sein könnte. Die Klage von Awilija und Munawwirum hätte sich demnach nicht gegen ihre beschenkte Tante Nin-kuzu gerichtet, sondern gegen die Erbengemeinschaft, welche bei der Teilung des Nachlasses von Nanna-meša diese Schenkung zu berücksichtigen hatte.

\subsection{Generation 7 im Familienzweig des Nanna-meša: Mannum-mēšu-lișșur und seine Brüder}

Als zentrale Gestalt der siebenten Generation erscheint Mannum-mēšu-lișșur. Das hat seinen Grund darin, dass die Urkunden, die ihn und seine Familie bezeugen, zum allergrößten Teil aus seinem Archiv stammen (s. Abschnitt 2). Mannum-mēšu-lișșur erhielt in der Teilung des Nachlasses seines Großvaters Nuska-amah anstelle seines bereits verstorbenen Vaters Awīlija einen Erbteil. Stone/ Owen (1991, 25. 88) setzten das Datum der entsprechenden Erbteilungsurkunde MC 3, Nr. 51 im Jahr Samsu-iluna 23 (1727) an. ${ }^{31}$ Damit hätte die Erbteilung untypischerweise zeitlich lange nach der umfangreichen Kauftätigkeit des Mannum-mēšu-lișșur in den Jahren Samsu-iluna 11-13 stattgefunden. Tatsächlich wurde die Erbteilungsurkunde MC 3, Nr. 51 aber im dritten Monat des Jahres Samsu-iluna 10 ausgestellt. ${ }^{32}$ Unmittelbar danach ist die Urkunde OECT 8, 16 (Si 10/03/23) anzusetzen, der zufolge Mannum-

31 Siehe Stone/Owen (1991, 88): 50 iti šeg gi $_{12}$ a mu sa-am-su-[i-luna lugal] 51á-kal ḩuš-r[a ...]. Damit meinten die Autoren offenbar das Jahr mu sa-am-su-i-lu-na lugal-e usu(Á.KAL) ĝ̀ir-ra den-líl-1e ... = Si 23. So auch im Neuabdruck der Textbearbeitung CUSAS 15, 23: u.E.2 iti šeg 12 $_{12}$ a mu sa-am-su-[i-lu-na lugal] ${ }^{3}$ u su ĝ̀ r - r [a ....].

32 Lies mit Foto und Kopie der Urkunde, Stone/Owen 1991, Pl. 28. S. 149: 50 iti šeg ${ }_{12}$-a mu sa-am-su-[i-[lu-na lugal] ${ }^{51}$ usu $\mathrm{mah}^{\star} \mathrm{d}^{*} \mathrm{~m}[\operatorname{arduk}(-\mathrm{ka}-\mathrm{ta})]$. 
mēšu-lișșur $12 \mathrm{~g}$ i $\hat{\mathrm{g}}_{4}\left(7,5 \mathrm{~m}^{2}\right)$ Wohnhaus aus seinem Erbteil und Vorzugsanteil gegen drei Pfründen seines Miterben Munawwirum, wohl größtenteils aus dessen Erbteil, tauschte. Erbteilung und teilweiser Tausch der Erbteile im Jahr Samsu-iluna 10 gingen also der regen Kauftätigkeit von Mannum-mēšu-lișșur in den Jahren Samsu-iluna 11-13 voraus.

Bereits vier Jahre vor Erhalt seines Erbteils war Mannum-mēšu-lișșur von Nannāja (Generation 6), dem Sohn des Sîn-iqišam, adoptiert worden. Die entsprechende Urkunde, MC 3, Nr. 27 (Si 6/07/-), ${ }^{33}$ dokumentiert die Eheschließung zwischen Nannāja, Sohn des Sîn-iqīšam, und Lamassum, Tochter des Ipqu-Ea, mit gleichzeitiger Adoption von vier Personen: außer Mannum-mēšu-lisșur auch Ninurta-muballiț, Sîn-māgir und Ina-Ekur-rabi, alle ohne Filiationsangabe. Es stellt sich die Frage, in welchem Verhältnis die vier adoptierten Personen zu der gleichzeitig von ihrem Adoptivvater Nannāja geehelichten Frau Lamassum standen.

Stone (1991, 22f.) stellte zwei Möglichkeiten zur Diskussion: 1) Die Adoptivsöhne Nannājas waren die leiblichen Kinder seiner neuen Frau Lamassum aus deren vorheriger Ehe. 2) Die Adoptivsöhne gehörten nicht zur Familie von Nannāja und seiner Frau Lamassum. Die erste Interpretationsmöglichkeit hielt Stone aus folgenden Gründen für weniger wahrscheinlich: In der Formulierung der Urkunde wird keine Verbindung hergestellt zwischen der Eheschließung und der Adoption, während in anderen Adoptionsurkunden dieser Art - den sogenannten ,type 3 adoptions" - die neue Ehefrau ihre Kinder dem Mann ausdrücklich zur Adoption geben würde. Stone/Owen (1991, 49-53) führen allerdings nur vier Urkunden der „type 3 adoptions“ auf, und nur in zweien ist der Adoptionspassus mit dieser Formulierung tatsächlich erhalten. Das sind zu wenige Belege, um davon auszugehen, dass eine Adoption von Kindern eines Ehepartners nur so formuliert sein kann. Als weiteres Argument gegen die leibliche Abstammung der adoptierten Kinder von Lamassum führte Stone $(1991,23)$ an: „that such an interpretation would not explain the complexities of interrelationship which are evidenced in the later texts“, allerdings ohne Nennung konkreter Belege.

Stone wollte eine Verwandtschaft der Adoptivsöhne Nannājas mit seiner geehelichten Frau Lamassum gern ausschließen, weil diese im Widerspruch zu ihrer Deutung der Adoptionen im Archiv des Mannum-mēšu-lișșur steht: Ihrer Ansicht nach wurden die Adoptionen aus

33 Als Grundlage für die folgende Diskussion dienen die Übersetzung von Westbrook $(1988,138)$ und die Bearbeitung von Obermark (1992, Part II, 159-161). Siehe auch Charpin (1994, 95b).
Bedarf an Vermögen seitens der Adoptierenden und ihrer Familien heraus getätigt. Mannum-mēšu-lișșur selbst sei wenig vermögend gewesen und habe sich adoptieren lassen, um Kapital zu erwerben und dieses für seinen sozialen Aufstieg zu nutzen. ${ }^{34}$

Zweifel an dieser Auffassung ruft allerdings schon der ansehnliche Erbteil des Mannum-mēšu-lișșur hervor, den er - wie gerade ausgeführt - bereits vor seinen zahlreichen Käufen im Jahr Samsu-iluna 10 erhielt. Auch die Eheund Adoptionsurkunde selbst spricht gegen die Deutung von Stone, wenn man sich die empfangenen Leistungen aller Parteien genauer ansieht: Nannāja brachte als zukünftige Erbschaft für seine Adoptivsöhne seinen eigenen Erbteil ein, bestehend aus Haus, Feld, Garten, Pfründen und beweglicher Habe, soviel vorhanden. ${ }^{35}$ Diesen Erbteil hatte er vermutlich erst kurz zuvor erhalten gemäß der Erbteilungsurkunde MC 3, Nr. 26 (Si 6?/05/-). ${ }^{36}$ Es ist nicht gesagt, dass den Adoptivsöhnen dieses Vermögen sofort zur Verfügung stand; wahrscheinlicher ist, dass sie es als eingesetzte Erben ihres Adoptivvaters erst nach dessen Ableben an sich nehmen konnten.

Unmittelbaren wirtschaftlichen Nutzen scheint nur der Ehemann und Adoptivvater Nannāja aus der Adoption gezogen zu haben: Er erhielt anscheinend von seiner Frau Lamassum und seinen vier Adoptivsöhnen eine Zahlung von 10 Schekeln Silber; leider ist der Text an dieser Stelle fragmentarisch. ${ }^{37}$ Entsprechend gibt es eine Strafklausel

34 Stone/Owen $(1991,19)$ : „... Mannum-mešu-lișșur, and to a lesser extent, his brother Ninurta-muballit were able to use the institution of adoption to place themselves in a powerful economic position. ... Mannum-mešu-lișșur's property ownership was only made possible through adoption. He was born of a property-less, but clearly wealthy, family, and apparently used adoption as a way of turning his family's wealth into an opportunity for social mobility. Since it was Mannummešu-lișșur's admission into the Sin-iqišam family (see fig. 2) that allowed him entry into the property-owning group, ....

35 MC 3, Nr.27: 9-12: ${ }^{9}$ é a-šà ĝeškiri ${ }_{6}$ gárza ${ }^{10}$ ù níĝ-gur ĝál-la a-na me-a-'bil 11 ka dub hुa-la-ba-š[è?] 12 i-in$\mathrm{ku}_{4}-\mathrm{re}-\mathrm{m}[\mathrm{e}-\mathrm{e} s \mathrm{~s}]$, „Haus, Feld, Garten, Pfründen und bewegliche Habe, soviel vorhanden, sind das, was er (= Adoptivvater Nannāja) ge[mäß] dem Wortlaut der Urkunde bezüglich (seines eigenen) Erbteils (in das Adoptionsverhältnis als Erbe für seine Adoptivsöhne) eingebracht hat." So mit Westbrook $(1988,138)$. Dieser las am Ende von Z. 11: h ha-1a-ba-[ni?]. Mit Blick auf Zeichenspuren (Stone/Owen 1991, Pl. 1: 11) und Grammatik erscheint eine Lesung ka dub ha la-ba-š [è?] etwas wahrscheinlicher. Anders Stone/Owen (1991, 63) und Obermark (1992, Part II, 159f.).

36 Zur Lesung des Jahresnamens dieser Urkunde s. Abschnitt 4.4.

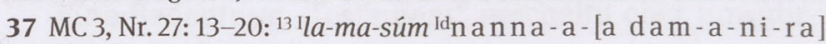
${ }_{14}$ Ima-an-nu-um-me-šu-l[i-șur] ${ }^{15}$ Id nin-urta-mu-ba-al-l[í-iț] ${ }^{16}$ Id sìn-ma[gir] 17 ù $i$-na-é-kur-[ra-bi] 18 Idnanna-a-a ad-[da-ne-ne-ra] $1910 \mathrm{gi}_{4} \hat{\mathrm{g}}_{4}$ 'kù'-b[abbar ...] ${ }^{20}[(\mathrm{x})]$ x [...], „Lamassum hat dem Nanna-aj[a, ihrem Ehemann], (und) Mannum-mēšu-lișșur, Ninurtamuballiț, Sîn-māgir und Ina-Ekur-rabi haben dem Nannāja, [ihrem] 
nur für Nannāja: Für den Fall, dass er das Ehe- und Adoptionsverhältnis beenden sollte, ging er des von ihm eingebrachten Vermögens verlustig und musste außerdem noch eine halbe Mine Silber zahlen. ${ }^{38}$ Wenn sich einer der Vertragspartner in einer wirtschaftlich schwächeren Position befunden haben sollte, dann war es - die Richtigkeit der Textkonstruktion vorausgesetzt - Nannāja, da er infolge der Adoption eine Zahlung erhielt und bei Vertragsbruch Strafe zahlen musste.

Interessant ist außerdem die Familienanbindung der vier adoptierten Männer. Zumindest zwei von ihnen gehörten schon vor der Adoption der weiteren Familie ihres Adoptivvaters Nannāja an. Als Vater des Mannum-mēšulișșur nennen spätere Urkunden Awilijija. ${ }^{39}$ Dass es sich dabei um Awīlija, den Sohn von Nuska-amah handelt, geht aus der gerade diskutierten Erbteilungsurkunde MC 3, Nr.51 (Si 10/03/-) hervor, gemäß der Mannummēšu-lișșur anstelle seines verstorbenen Vaters Awilija mit seinem Onkel Munawwirum den Nachlass seines Großvaters Nuska-amah teilte. Akzeptiert man das vermutete Sohnschaftsverhältnis des Sîn-iqišam zu Addakalla (s. Abschnitt 3.2. und 4.2.), dann haben Awilija und Nannāja denselben Urgroßvater Lu-Bau, sind also Cousins zweiten Grades. Für Awīlijas Sohn, Mannum-mēšu-lișșur, ist Nannāja ein Onkel dritten Grades.

Ninurta-muballit ist der Bruder des Mannum-mēšulișșur. Er tritt in der Urkunde BE 6/2, 64 (Si 29/02/20) ge-

Va[ter], 10 Schekel Sil[ber ...]“ (danach abgebrochen). So mit Westbrook $(1988,138)$ und Obermark (1992, Part II, 159f.). Stone/Owen (1991, 63f.) sahen Frau und Kinder als Empfänger der Zahlung, berücksichtigten aber nicht, dass in Vs. 13 nach Idn a n na-a-[a] Platz für weitere Zeichen war.

38 MC 3, Nr. 27 Rs. $2^{\prime}-10^{\prime}: z^{\prime}$ tuk[um-bi dnanna-a-a] ${ }^{\prime}$ Ila-ma[súm dam-a-ni-ra] 4' Ima-an-nu-um-me-[šu-li-șur] 5' Idnin-urta-

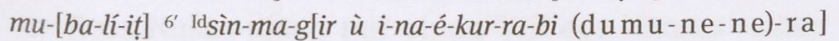
$7^{\prime}$ dam- $\hat{g} u_{10}$ nu-me-en d[umu-ĝu $u_{10}$ nu-me-en] ${ }^{8}$ ba-an$n a-d u_{11}$ é [a-šà geškiri $i_{6}$ gárza] ${ }^{9}$ ba-ra- $e_{11}$-[dè] ${ }^{10^{\prime}}$ ù $1 / 2$ ma-na kù-'babbar' [ì-lá-e], „We[nn Nannāja] zu Lama[ssum, seiner Ehefrau], (und) [zu] Mannum-mē[šu-lișșur], Ninurta-mu[balliț], Sînmāg[ir und Ina-Ekur-rabi, (seinen Söhnen)], sagen sollte: ,Du bist nicht meine Frau; [ihr seid nicht meine] S[öhne!]', dann wird er des Hauses, [Feldes, Gartens und der Pfründe] verlustig gehen und $1 / 2$ Mine Silber [zahlen]. “ So mit Westbrook $(1988,138)$ und Obermark (1992, Part II, 159f.). Stone/Owen $(1991,64)$ gingen davon aus, dass die Strafe den Adoptivsöhnen angedroht wurde, berücksichtigten aber nicht, dass in Rs. $2^{\prime}$ nach tu k [u m-bi] noch viel Platz ist. AuBerdem kann der Sprecher der Aussage „Du bist nicht meine Ehefrau!" nur der Ehemann sein und gegen Stone/Owen nicht die Adoptivsöhne, s. dazu bereits Charpin (1994, 95b).

39 Stone/Owen (1991), Nr. 28: 6f., Nr.34: 6f., Nr. 35: 9f., Nr. 37: 5'f., Nr. 38: 8 f., Nr. 39: 8, Nr.40: 9f., Nr. 41: 7 f., Nr. 42: 7 f., Nr. 43: 5f., Nr. 44: 9 f., Nr. 45: 12f., Nr. 47: 7'f., Nr. 48: 7 f., Nr. 49: 10f., Nr. 50: 7 f. (Käufe); Nr. 51: Siegel (Erbteilung), Nr. 30: Siegel (Tausch), Nr. 31: $1 \mathrm{f}$. (Prozess), Nr. 52: 5 (Rückkauf). meinsam mit Sohn und Ehefrau des offenbar inzwischen verstorbenen Mannum-mēšu-lișșur als Verkäufer eines Grundstückes auf, das Mannum-mēšu-lișșur zu Lebzeiten erworben hatte. ${ }^{40}$ Die Filiation weist ihn ebenso wie Mannum-mēšu-lișșur als Sohn des Awilīja aus. Ein Mann namens Ninurta-muballiț, Sohn von Awilija, ist auch belegt in OECT 8, 21 (Si 11/[...]/22). Er wird dort adoptiert durch Ilī-ippalsam, Sohn von Sîn-māgir, und seine Frau Elmēšum. Auf den ersten Blick erscheint es fraglich, ob dieser Ninurta-muballit mit dem Bruder des Mannummēšu-lișșur identisch ist, der fünf Jahre zuvor, im Jahr Samsu-iluna 6, von Nannāja adoptiert worden war. Die Namen der Zeugen machen jedoch die Zugehörigkeit von OECT 8, 21 zum Archiv des Mannum-mēšu-lișșur wahrscheinlich: Vier der vermutlich insgesamt sechs Zeugennamen sind erhalten und alle vier kommen auch sonst mehrfach in Urkunden der Familie des Mannum-mēšulișṣur vor. ${ }^{41}$ Entweder also ist OECT 8, 21 der erste altbabylonische Beleg dafür, dass ein Individuum gleichzeitig von mehreren verschiedenen Personen adoptiert sein konnte, ${ }^{42}$ oder man muss davon ausgehen, dass Nannāja,

40 Für Überlegungen zu den rechtlichen Hintergründen des gemeinsamen Auftretens von Ehefrau bzw. Bruder eines Verstorbenen und dessen Erben als Verkäufer, speziell auch in Hinsicht auf BE 6/2, 64, s. Meinhold (2014, 23-27).

41 Lú-Enlila, Sohn von É-lú-ti, Verwalter (a g rig) des Enlil: OECT 8, 19: 21, OECT 8, 11: 23, OECT 8, 16: 17, OECT 8, 1: 18, BE 6/2, 38: 20, OECT 8, 2: 19, OECT 8, 9: 19, OECT 8, 10: 23, OECT 8, 7: 19, OECT 8, 8: 19, BE 6/2, 64: 21 (Zeuge; zumeist an erster Stelle genannt). Nuskanī̌u und sein Bruder Ilī-ippalsam, Söhne von Ninurta-mušallim: OECT 8, 9: 20 f. (Zeugen; wie in OECT 8, 21 nach Lu-Enlila genannt; Schreiber ist Apil-ilišu, evtl. Sohn von Nuska-nīšu s. BE 6/2, 41: 22'). Nuska-nišu ist evtl. auch Zeuge in OECT 8, 1: 19, dort allerdings ohne Filiation, aber mit Titel du b - sar. Rīš-Ea, Sohn von Sînātum: OECT 8, 6: 19, MC 3, Nr. 37: 19' (Zeuge), OECT 8, 1: 3 (Nachbar von gekauftem Wohnhaus des Mannum-mēšu-lișșur). Vgl. auch die Belege für diese Zeugen außerhalb des Archivs des Mannum-mēšu-lișșur: Lu-Enlila: BE 6/2, 41: 19' (Zeuge), SAOC 44, 89 Rs. 7 (Zeuge; Archiv des Attā). Nuska-nǐšu und Ilī-ippalsam: BE 6/2, 41 (Verkäufer bzw. Zeuge), UM 29-16-672 (Erben).

42 Van De Mieroop (1991-1993, 127b) vermutete eine solche Mehrfachadoption auch für Mannum-mēšu-lisșur. Dieser wurde nachweislich im Jahr Samsu-iluna 6 adoptiert durch Nannāja, den Sohn von Sîn-iqišam, s. MC 3, Nr. 27. Mit Van De Mieroop könnte er auBerdem ein Adoptivsohn von Nannājas Bruder Ibbi-Enlil gewesen sein, da er diesen mit Getreide versorgte, s. OECT 8, 11 (Si 10/09/03, s. Stone/Owen 1991, Nr. 31). Ein Adoptionsverhältnis ist in letzterem Beleg allerdings nicht erwähnt. Dass ein solches bestand, ist m.E. eher unwahrscheinlich, da Mannum-mēšu-lișșur die Kosten des Getreides für Ibbi-Enlil von dessen Söhnen zurückforderte. Mit der Versorgung Ibbi-Enlils erfüllte er also nicht unentgeltliche (Adoptiv-) Sohnespflichten, sondern er gewährte Ibbi-Enlil eine Art Darlehen, das er nach dessen Ableben von seinen Erben zurückfordern konnte. 
der frühere Adoptivvater des Ninurta-muballiț, zwischenzeitlich verstorben war.

Für die dritte und vierte Person, die Nannāja gemäß MC 3, Nr. 27 adoptierte, Sîn-māgir und Ina-Ekur-rabi, sind bislang keine weiteren Belege bekannt. ${ }^{43}$

Zusammenfassend erscheinen die Argumente Stone's gegen eine leibliche Abstammung der vier Adoptivsöhne von der geehelichten Frau Lamassum kaum überzeugend, denn: 1) Der Formulierung der Urkunde und dem Fehlen eines Hinweises auf eine Verbindung zwischen EheschlieBung und Adoption kann man nicht allzu viel Gewicht beimessen, da es nur wenige vergleichbare Urkunden gibt. 2) Das Motiv für die Adoption war wohl kaum Bedarf an Vermögen seitens der Adoptivsöhne. Wenn jemand wirtschaftlich von der Adoption profitierte, so war es der Adoptivvater Nannāja. 3) Zumindest zwei der vier adoptierten Personen sind nachweislich Brüder und wahrscheinlich mit dem Adoptivvater verwandt. 4) Die Annahme einer leiblichen Abstammung der vier adoptierten Personen von Lamassum steht mit den Verwandtschaftsbeziehungen, wie sie aus anderen Urkunden bekannt sind, keineswegs im Widerspruch, sondern es ergibt sich folgendes, stimmiges Bild: ${ }^{44}$ Lamassum war vor dem Jahr Samsu-iluna 6 mit Awilijja, Sohn von Nuska-amah, verheiratet. Beide hatten vier Söhne: Mannum-mēšu-lișșur, Ninurta-muballiț, Sînmāgir und Ina-Ekur-rabi. Nachdem Awilija gestorben war, nahm sein mutmaßlicher Cousin zweiten Grades, Nannāja, die Lamassum zur Frau. Motivation dafür war vielleicht der Wohlstand dieses Familienzweigs, von dem Nannāja sich einen Anteil erhoffte und ihn in Form von 10 Schekeln Silber wohl auch gleich bekam.

Schwierigkeiten in dieser Interpretation bereitet allerdings die Erbteilungsurkunde MC 3, Nr. 51 (Si 10/03/-). Sie nennt nur zwei Erben des Nuska-amah: seinen Enkel Mannum-mēšu-lișșur, der den Erbteil seines verstorbenen Vaters Awilìja entgegennahm, und seinen Sohn $\mathrm{Mu}-$

43 Stone/Owen (1991, 20. 23) erwogen für Sîn-māgir eine Identität mit dem Zeugen Sîn-māgir, Sohn des Sîn-iqišam, in MC 3, Nr. 26 iv 4-5, den sie als Bruder von Nannāja und Ibbi-Enlil ansetzten. Dass jedoch Nannāja seinen eigenen Bruder adoptiert haben sollte, ist m. E. äußerst unwahrscheinlich. Die Personennamen Sîn-māgir und Sîn-iqišam sind in Nippur sehr häufig; Gleichnamigkeit ist deshalb kein ausreichendes Argument für Identität.

44 So mit Westbrook (1988, 63a), Van De Mieroop (1991-1993, 127b zu Text 27 und 128a-b) und Charpin (1994, 95b zu Nr. 27). Z. B. Westbrook (1988, 63a): „Although not expressly stated, it is clear from the context that the four are the children of the wife. In the only extant penalty-clause, they are ranged with the wife against the husband, who is contemplated as divorcing and repudiating the adoption in the same action. They also appear in a broken clause to be jointly making some cash payment to the husband." Auch Obermark (1992, Part I, 22 und 28, Anm. 22) rechnet YOS 15, 73 zu den type 3 adoptions. nawwirum. Wenn alle vier Adoptivsöhne des Nannāja leibliche Söhne von Awilija, Sohn von Nuska-amah, und Lamassum waren, warum erscheint dann nur einer von ihnen, Mannum-mēšu-lișșur, als Erbe an seines Vaters statt?

Diese Frage stellt sich allerdings auch, wenn man wie Stone - annimmt, dass zwei der Adoptivsöhne, Sînmāgir und Ina-Ekur-rabi, Außenseiter waren und nicht mit den anderen Familienmitgliedern verwandt: Denn zumindest Ninurta-muballit ist, wie gerade dargelegt, als Bruder von Mannum-mēšu-lișșur anzusehen, und zumindest er sollte daher als Erbnehmer am Erbteil seines verstorbenen Vaters in der Erbteilungsurkunde erwähnt sein. Es wäre denkbar, dass Mannum-mēšu-lișșur stellvertretend für seine Brüder den Erbteil seines Vaters entgegennahm. Doch tauschte er gleich darauf das in seinem Erbteil und Vorzugsanteil vorhandene Haus gegen Pfründen seines Onkels und Miterben Munawwirum, was nahelegt, dass er über den gesamten Erbteil frei verfügen durfte und ihn nicht mit seinen mutmaßlichen Brüdern teilen musste. Die Frage, warum Ninurta-muballiț, Sîn-māgir und InaEkur-rabi als Söhne des Awìlija nicht in der Erbteilungsurkunde erwähnt sind, bleibt vorerst offen.

\subsection{Generation 8 im Familienzweig des Nanna-meša: Mannum-mēšu-lișșurs Sohn, Iddin-Ištar}

Mannum-mēšu-lișșur hatte zusammen mit seiner Ehefrau Narubtum einen Sohn namens Iddin-Ištar. Das geht aus der jüngsten Urkunde des Archivs, BE 6/2, 64 (Si 29/02/20), hervor. Iddin-Ištar war vermutlich der einzige Sohn Mannum-mēšu-lișșurs, s. dazu ausführlich Abschnitt 4.6.

\section{Die Entwicklung des Familienvermögens}

Der folgende Abschnitt vergleicht die Erbteilungsurkunden im Archiv des Mannum-mēšu-lișșur miteinander vor dem Hintergrund des rekonstruierten Stammbaums (s. Anhang 2). Zu beachten ist, dass Erbteilungsurkunden nicht in jedem Fall den gesamten Nachlass eines Erblassers enthalten müssen, da manche Güter über lange Zeit in Erbengemeinschaft verwaltet wurden. Das Vermögen eines Erblassers und seiner Familie konnte unter Umständen größer sein als in einer Erbteilungsurkunde dokumentiert. 


\subsection{Die Erbteilungen im Familienzweig des Nanna-meša bis Generation 5}

Im Familienzweig des Nanna-meša sind der Erbteil Nannamešas selbst (Generation 4) sowie der Nachlass seines Sohnes, Nuska-amah (Generation 5), bekannt. ${ }^{45}$ Der zeitliche Abstand zwischen beiden Erbteilungen beträgt ca. 97 Jahre. ${ }^{46}$ Nuska-amah war aber schon lange vor der Teilung seines Nachlasses verstorben, s. Abschnitt 3.3. Sein Nachlass wurde offenbar über mindestens 22 Jahre hinweg in Erbengemeinschaft verwaltet, zunächst von seinen Söhnen Awīlija und Munawwirum und nach dem Tod des Awilīja von dessen ältestem Sohn Mannum-mēšulișșur und Munawwirum. Zwischen der Teilung des Nachlasses von Lu-Bau und derjenigen von Nuska-amah fehlt uns als Bindeglied leider die Erbteilungsurkunde bezüglich des Nachlasses von Nanna-meša. Trotzdem ist es aufschlussreich, den Erbteil, den Nanna-meša erhielt, dem gesamten Nachlass, den sein Sohn Nuska-amah weitervererbte, gegenüberzustellen (siehe Tabelle 2 auf folgender Seite).

Der Erbteil von Nanna-meša enthält Wohnhaus, unbebautes Hausgrundstück, Türen, Feld, Pfründen, Garten und Mobilien, der Nachlass des Nuska-amah hingegen Wohnhaus, Pfründen und einen zà-gu-la-Tisch. Das bedeutet nicht unbedingt, dass Nuska-amah nicht mehr besessen haben könnte. Vielleicht behandelt die Erbteilungsurkunde nur einen Teil seines gesamten Besitzes. Deshalb ist nur die Schnittmenge zwischen dem Erbteil von Nanna-meša und dem Nachlass von Nuska-amah vergleichbar. Nuska-amah verfügte nur über einen Teil des von seinem Vater Nanna-meša ererbten Wohnhauses, aber über alle Pfründen aus dem Erbteil seines Vaters in annähernd voller Höhe und zusätzlich noch über vier weitere.

Dass Nuska-amah nur einen Teil des Hauses seines Vaters Nanna-meša weitervererbte, ist verständlich, denn die oben vorgeschlagene Interpretation der Klageverzichturkunde OECT 8, 4 legt nahe, dass Nuska-amah den Besitz seines Vaters mit drei Brüdern und einer Schwester teilen musste: Die Schwester Nin-kuzu bekam vom Vater Nanna-meša zu dessen Lebzeiten $15 \mathrm{gi} \hat{\mathrm{g}}_{4}\left(9 \mathrm{~m}^{2}\right)$ Wohnhaus geschenkt. Vorausgesetzt, diese $15 \mathrm{gi}_{4}$ stammten aus Nanna-mešas ererbten $80 \mathrm{gi}_{4}\left(48 \mathrm{~m}^{2}\right)$, so verringerte sich sein ererbter Hausbesitz auf $65 \mathrm{gi} \hat{\mathrm{g}}_{4}\left(39 \mathrm{~m}^{2}\right)$.

45 Erbteil Nanna-mešas in der Teilung des Nachlasses von Lu-Bau: OECT 8, 17: 1-23 // OECT 8, 18: 1-20 (Sîn-iqī̌šam 3 oder 4/12/-; s. Stone/ Owen 1991, Nr. 24 // Nr. 53; für Lu-Bau als Erblasser s. Abschnitt 3.1). Nachlass Nuska-amahs: MC 3, Nr. 51 (Si 10/03/-).

46 Auf Grundlage des „Tableau synchronique Isin-Larsa-Babylone“ von Charpin (2004, 385-387).
Teilt man dies unter vier Erben auf, so erhält man vier Erbteile zu je rund 14,5 $\mathrm{g} \mathrm{i} \hat{\mathrm{g}}_{4}$ und einen Vorzugsanteil von $10 \% \mathrm{zu} 6,5 \mathrm{gi}_{4}$ für den ältesten Erben. Der älteste Erbe hätte nach dieser Rechnung also insgesamt $21 \mathrm{~g}$ i $\hat{\mathrm{g}}_{4}(12,6$ $\left.\mathrm{m}^{2}\right)$ bekommen, was sehr nahe an den $22 \mathrm{gi} \hat{\mathrm{g}}_{4}\left(13,2 \mathrm{~m}^{2}\right)$ Wohnhaus liegt, die Nuska-amah weitervererbte. Auch andere Szenarien wären denkbar, denn wir wissen nicht, ob Nuska-amah tatsächlich der älteste Sohn Nanna-mešas war, dem der Vorzugsanteil zustand, ${ }^{47}$ oder ob er oder Nanna-meša weitere Wohnhausflächen zu ihren ererbten hinzukauften.

Geht man davon aus, dass Nuska-amah den väterlichen Besitz an Wohnhaus mit seinen Geschwistern teilen musste, dann wundert es, dass er dieselben Pfründen in ziemlich genau demselben Umfang, wie von seinem Vater ererbt, an seine Nachkommen weitergab. Zwei Erklärungen dafür sind denkbar: Entweder hatte Nuska-amah nur einen Teil der Pfründen über sein Erbe erhalten und die übrigen nach und nach seinen Brüdern und Miterben abgekauft oder die Pfründen waren bei der Teilung des Nachlasses des Nanna-meša allein an Nuska-amah gegangen. Im letzteren Fall wäre anzunehmen, dass Nuska-amah der älteste Sohn von Nanna-meša war, die Pfründen als Vorzugsanteil erhielt und seine Miterben durch Ausgleichszahlungen kompensierte, ein Vorgehen, das gelegentlich auch in anderen Familien in Nippur zu beobachten ist. ${ }^{48}$

\subsection{Die Erbteilungen im Familienzweig des Adda-kalla bis Generation 5}

Für den Familienzweig des Adda-kalla, Sohn von Lu-Bau, liegt eine ähnliche Quellenlage vor wie für den Familienzweig seines Bruders Nanna-meša: Wir kennen aus der Erbteilungsurkunde bezüglich des Nachlasses von Lu-Bau (Generation 3) den Erbteil von Adda-kalla, haben keine Erbteilungsurkunde seines Nachlasses, sondern erst wieder eine Erbteilungsurkunde bezüglich des Nachlasses von Adda-kallas mutmaßlichem Sohn Sîn-iqišam (Gene-

47 Der zà-gu-la-Tisch in seinem Nachlass (MC 3, Nr. 51: 11, s. Anhang 1) kann nicht als Hinweis darauf gewertet werden, denn es vererbten auch jüngere Söhne Tische dieser Art an ihre jeweils ältesten Söhne. So findet sich z. B. ein zà-gu//gú - la -Tisch im Vorzugsanteil des älteren Sohnes des Bìtūa, TIM 4, 8: 2 // TIM 4, 16: 2, aber auch im Nachlass seines jüngeren Sohnes, TIM 4, 4: 4 // O’Callaghan (1954) 142, NBC 8935: 4; ebenso im Vorzugsanteil des ältesten Sohnes des Imgūa, TIM 4, 1: 5, und im Nachlass eines seiner jüngeren Söhne, TIM 4, 2: 5 // TIM 4, 3: 5. Vgl. auch den zà-gu-la-Tisch in PBS 8/1, 12: 4, einer Erbteilung des Nachlasses eines jüngeren Sohnes von Abbakalla, Sohn des Ninlil-ziĝu.

48 Z. B. BE 6/2, 26; BE 6/2, 43 und weitere Erbteilungen in der Familie des Ninlil-ziĝu, s. A. Goddeeris, TMH 10 (in Vorbereitung). 
Tabelle 2: Der Erbteil Nanna-mešas und der Nachlass seines Sohnes Nuska-amah

\section{Geteilte \\ Besitztümer}

\section{Erbteil des Nanna-meša}

aus dem Nachlass seines

Vaters Lu-Bau,

OECT 8, 17: 1-23 // 18: 1-20

(Sîn-iqiišam 3 oder 4/12/-)

80 gi $_{4}$ é dù-a

2 Grundstücke à 3 bzw. 4 sar kislah

3 verschiedene Türen

1 iku a-šà uz-za

60 sar a-šà uz-za ki-duru

2 iku a-šà $i_{7}$-ĝ̀iri-da//ta-bala-e

Pfründen:

nam-ì-du ${ }_{8}$ ká sumun den-líl-lá

nam-ì-du ${ }_{8}$ ká gu-la

nam-ì-du 8 (ká) é-gal mah

nam-ì-du ${ }_{8}$ ká//é ús-gíd-da

nam-ì-du 8 ká gal dnin-líl-lá

nam-ì-du 8 ká ĝeš-kíĝ-ti

nam-ì-du 8 ká dnin-ĝá-ge ${ }_{4}^{-a}$

nam-ì-du ${ }_{8}$ ká//é du -kù

nam-é-da-di (den-líl dnin-líl)

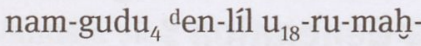

an-ki

na[m-ì-du ${ }_{8}$ kisal $]$ dnin-líl

[nam-x x x (x) l]a-ma-sà-tum

nam-ì-du 8 ká níĝ-ur ${ }_{5}$-ra

Garten

Mobilien

zà-gu-la-Tisch

\section{2,5 Tage/Jahr}

$-$

22,5 Tage/Jahr (nur in OECT 8,17 )

22,5 Tage/Jahr (nur in OECT 8, 17)

22,5 Tage/Jahr

22,5 Tage/Jahr

11,5 Tage/Jahr

22,5 Tage/Jahr

180 ? Tage/Jahr ${ }^{49}$ (nur in OECT 8,17 )

$-$$$
-
$$$$
-
$$$$
-
$$

Hälfte eines Gartens

aus dem Erbteil des Ur-Lumma

1 Mörser, 3 Wagenteile
Gesamter Nachlass des Nuska-amah, Sohn von Nanna-meša,

MC 3, Nr. 51, s. Neubearbeitung

Anhang 1

(Samsu-iluna 10/03/-)

[2]2 gig $\hat{g}_{4}$ é dù-a

$-$

$-$

$-$

$-$

2[2(,5)?] Tage/Jahr

2[2(,5)?] Tage/Jahr

2[2(,5)?] Tage/Jahr

2[2(,5)?] Tage/Jahr

22 Tage/Jahr

11 Tage/Jahr

22,5 Tage/Jahr

180 Tage/Jahr

40 Tage/Jahr

2,5 Tage/Jahr

5 Tage/Jahr

24 Tage/Jahr

$-$

$-$

1
49 Angaben zu dieser Pfründe stehen in OECT 8, 17: 18. 40 (s. Stone/ Owen 1991, Nr. 24). Während man in Z. 18 unter Umständen mu-à m 'iti? ? 6-kam lesen kann, steht in Z. 40: $\mathrm{mu}$-àm $\mathrm{u}_{4}$ 6-ka m, so nach Foto Stone/Owen (1991) Pl. $54 \mathrm{f}$. Angesichts der Tatsache, dass sich im Nachlass des Nuska-amah aber 6 Monate dieser Pfründe befinden (s. Anhang 1, Cornell 23: 9. 22f. 39 f.: $18+81+[60]+21$
= 180 Tage), und im Nachlass des Sîn-iqiǐam ebenfalls 6 Monate (MC 3, Nr. 26 i 1 f. i 17 f. ii 11 f.: [18] + 81 + 81= 180 Tage), muss der Nachlass des Lu-Bau wohl ein ganzes Jahr é-da-di-Pfründe umfasst haben, von dem jeder seiner Söhne, die Hälfte, also 6 Monate $=180$ Tage bekam; entsprechend wäre in OECT 8, 17: 18 und 40: mu-àm iti 6-ka m zu erwarten. 
Tabelle 3: Der Erbteil Adda-kallas und der Nachlass seines mutmaßlichen Sohnes Sîn-iqǐ̌sam

\section{Geteilte \\ Besitztümer}

\section{Erbteil des Adda-kalla aus dem Nachlass seines Vaters Lu-Bau,}

OECT 8, 17 // OECT 8, 18

(Sîn-iqišam 3 oder 4/12/-)

\section{Wohnhaus}

unbebautes Hausgrundstück

Türen

Feld

$$
\begin{aligned}
& \text { nam-ì-du }{ }_{8} \text { ká ĝeš-kíĝ-ti } \\
& \text { nam-ì-du }{ }_{8} \text { ká dnin-ĝá-ge }{ }_{4}^{-a} \\
& \text { nam-ì-du } 8 \text { ká du }{ }_{6} \text {-kù(-ga) } \\
& \text { nam-é-da-di (den-líl dnin-líl) } \\
& \text { Garten }
\end{aligned}
$$

$$
80 \text { gi }_{4} \text { é dù-a }
$$

2 Grundstücke zu 3 bzw. 4 sar kislah

3 verschiedene Türen

100 sar a-šà uz-za

60 sar a-šà uz-za ki-duru 200 sar a-šà $i_{7}$-ĝ̀iri-da//ta-bala-e

$$
\begin{aligned}
& \text { 22,5 Tage/Jahr } \\
& \text { 22,5 Tage/Jahr (nur in OECT 8, 17) } \\
& \text { 22,5 Tage/Jahr (nur in OECT 8, 17) } \\
& \text { 22,5 Tage/Jahr }
\end{aligned}
$$

22,5 Tage/Jahr

11,5 Tage/Jahr

22,5 Tage/Jahr

180? Tage/Jahr50 (nur in OECT 8, 17)

Hälfte eines Gartens

aus dem Erbe des Ur-Lumma
Gesamter Nachlass des Sîn-iqišam, Sohn von Adda-kalla, MC 3, Nr. 26

(Si 6?/05/-)

80 gig $_{4}$ é dù-a

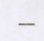

$-$

160 sar a-šà uz-za

180 sar a-šà $i_{7}$-ĝ̀iri-ta-bala-e

$-$

$2[2,5$ ? $]$ Tage/Jahr $([2,5 ?]+10+10)$

\section{2,5 Tage/Jahr}

nam-ì-du 8 é ús-gíd-gíd-da ù ká gal

dnin-líl-lá

22,5 Tage/Jahr

14 Tage/Jahr

7 Tage/Jahr $(0+[3,5]+3,5)$

180 Tage/Jahr ([18]+81+81)

Mobilien

4 Wagenteile, 1 Stuhl

ration 5). Hinzu kommt eine Urkunde über die Teilung des Nachlasses von Sîn-iqī̌ams Sohn Ibbi-Enlil (Generation 6). Aufschlussreich ist auch hier zunächst eine Gegenüberstellung des Erbteils von Adda-kalla, Sohn von Lu-Bau, und des gesamten Nachlasses seines vermuteten Sohnes Sîn-iqī̌am (siehe Tabelle 3).

Sowohl im Erbteil von Adda-kalla als auch im Nachlass von Sîn-iqišam kommen Wohnhaus, Felder und Pfründen vor. Es gibt kleinere Abweichungen: So vererbt Sîn-iqiišam von einem Feld 20 sar weniger als sein Vater geerbt hatte ( $\mathrm{a}$-šà $i_{7}-\hat{g}$ ìr i-da//ta-bala-e), von einer Pfründe vererbt er 15,5 Tage weniger ( $n a m-i ̀-d u_{8}$ ká $\mathrm{du}_{6}$-kù-ga), von einer anderen Pfründe 2,5 Tage mehr (nam-ì-du $u_{8}$ ká dnin-ĝá-ge $e_{4}^{-a}$ ). Zwei Pfründen, un-

50 Siehe Anm. 49. bebautes Hausgrundstück, Türen, Garten und Mobilien, die Adda-kalla geerbt hatte, sind in der Erbteilungsurkunde bezüglich des Nachlasses von Sîn-iqī̌sam nicht erwähnt. Abgesehen von diesen Abweichungen und einer z. T. etwas anderen Reihenfolge der aufgelisteten Besitztümer kommt der Nachlass von Sîn-iqī̌sam aber dem von Adda-kalla ererbten Besitz hinsichtlich Art und Menge sehr nahe, was Stone/Owen $(1991,20.29)$ zu der berechtigten Annahme veranlasste, Sîn-iqǐ̌šm sei ein Sohn Adda-kallas (s. Abschnitt 3.2).

Wie aber kommt es zu der Übereinstimmung zwischen dem Erbteil von Adda-kalla und dem Nachlass seines mutmaßlichen Sohnes Sîn-iqišam? Hatte Sîn-iqišam allein das gesamte Erbe von Adda-kalla übernommen? ${ }^{51}$ Dass 
dem nicht so ist, erfahren wir aus der Erbteilungsurkunde selbst. Zwischen den Erbteilen und der Teilungsklausel findet sich folgender Einschub, MC 3, Nr. 26 Rs. iii 7-20 (z. T. anders Stone/Owen 1991, 61f.):

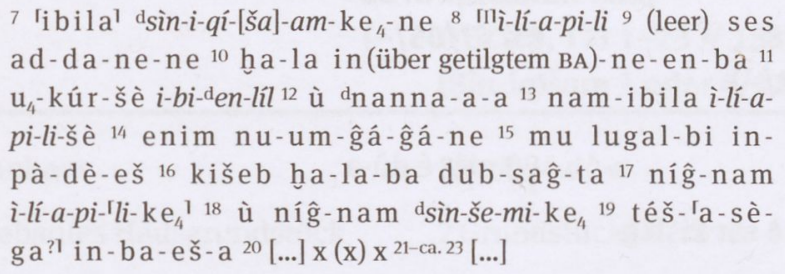
ad-da-ne-ne ${ }^{10}$ ha-la in (über getilgtem BA)-ne-en-ba ${ }^{11}$ $\mathrm{u}_{4}$-kúr-šè $i$-bi-den-líl ${ }^{12}$ ù ${ }^{\mathrm{d}} \mathrm{n}$ anna-a-a ${ }^{13} \mathrm{nam}$-ibila i-lí-api-li-šè ${ }^{14}$ enim nu-um-ĝá-ĝá-ne ${ }^{15} \mathrm{mu}$ lugal-bi inpà-dè-eš ${ }^{16}$ kišeb ha-la-ba dub-sâg-ta ${ }^{17}$ níğ-nam

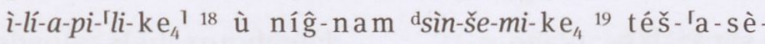
$\mathrm{ga}^{?}$ in - ba-e š-a ${ }^{20}[\ldots] \mathrm{x}(\mathrm{x}) \mathrm{x}^{21-\mathrm{ca} .23}[\ldots]$

„Den Erben von Sîn-iqišam hat Ilī-āpilī, der Bruder ihres Vaters, sein ererbtes Vermögen zugeteilt. In Zukunft werden Ibbi-Enlil und Nannāja keinen Anspruch auf den Status als Erben des Ilī-âpilī erheben; das haben sie mit einem Eid beim König geschworen. Entsprechend? der früheren Erbteilungsurkunde, gemäß? der sie den gesamten Besitz von Ilì-āpilī und den gesamten Besitz von Sîn-šēmi miteinander geteilt haben, ...“

Das Verständnis des letzten Satzes ${ }^{52}$ wird erschwert durch die folgende Lücke; außerdem bleibt unklar, wer Sîn-šēmi ist. ${ }^{53}$ Aus den beiden voranstehenden Sätzen geht jedoch hervor, dass Ilī-āpilī, der Bruder des Erblassers Sîniqišam, seinen Neffen, den Söhnen und Erben des Sîniqǐ̌šm, seinen ererbten Besitz vermachte. Es hatte also eine Teilung des Nachlasses von Adda-kalla unter seinen mutmaßlichen Söhnen Sîn-iqišam und Ilī-āpilī gegeben. Anlässlich der Teilung des Nachlasses von Sîn-iqišam wurde dieser Besitz durch Schenkung des Erbes von Ilīāpilī an die Erben des Sîn-iqī̌sam wieder zusammengeführt. Die Erben des Sîn-iqī̌am, Ibbi-Enlil und Nannāja, leisteten Klageverzicht auf einen „Status als Erben“ des Ilī-āpilī (nam-ibila). Gemeint ist damit wohl, dass sie aus dem dokumentierten Vermächtnis keinen Erbanspruch gegenüber ihrem Onkel, Ilī-āpilī, und seinem zukünftigen Nachlass, d. h. seinem Besitz über sein ererbtes Vermögen hinaus, ableiten durften.

Die weitgehende Entsprechung zwischen dem Erbteil des Adda-kalla und dem Nachlass seines mutmaßlichen Sohnes Sîn-iqǐ̌̌m zuzüglich des ererbten Vermögens von Ilī-āpilī zeigt, dass im Familienzweig des Adda-kalla ebenso wie in demjenigen des Nanna-meša der von Lu-Bau ererbte Besitz mit nur geringen Verlusten über gut 90 Jahre recht konstant erhalten blieb. Das gilt zumindest für alle Posten, die in den jüngeren Erbteilungsurkunden beider Familienzweige aufgeführt sind. Aus dem Umstand,

52 Für Hinweise zu Lesung und Deutung danke ich W. Sallaberger. 53 Sîn-šēmi trat ebenso wie Ilì-āpilì auch in MC 3, Nr. 27 Rs. $11^{\prime} .16^{\prime}$ als Zeuge auf. Mit ihrer Zeugenschaft demonstrierten beide ihr Einverständnis damit, dass Nannāja seinen z. T. von ihnen ererbten Besitz seinen Adoptivsöhnen überschrieb. dass die jüngeren Erbteilungsurkunden manche Posten aus dem Nachlass des Lu-Bau nicht aufführen, kann man nicht unbedingt einen Verlust von Familienbesitz folgern, da immer die Möglichkeit besteht, dass diese Posten ungeteilt in Erbengemeinschaft verwaltet wurden oder in Erbteilungsurkunden geteilt wurden, die nicht auf uns gekommen sind.

\subsection{Vergleich der Entwicklung des vererbten Familienvermögens in beiden Familien- zweigen bis Generation 5}

Veranschaulichen lässt sich die nahezu konstante Erhaltung des Familienbesitzes bis zur Generation 5 durch eine Gegenüberstellung der Erbteile der ältesten Erben der Generation 6 in den Familienzweigen von Nanna-meša und Adda-kalla, Ibbi-Enlil und Awilija (siehe Tabelle 4). Da letzterer zum Zeitpunkt der Erbteilung bereits verstorben war, erhielt sein ältester Sohn Mannum-mēšu-lișșur (Generation 7) seinen Erbteil. ${ }^{54}$

Mannum-mēšu-lișșur erhielt zwar mehr Pfründen, insgesamt 68 Tage mehr an verschiedenen Pfründen, dafür bekam er weniger Haus, nur gut ein Viertel von dem, was Ibbi-Enlil erbte, und kein Feld. An Wert könnten sich die Erbteile der beiden ältesten Erben in den dokumentierten Erbteilungen der Generation 6 in etwa entsprechen.

\subsection{Die Entwicklung des Familienvermögens in den Generationen 6 bis 8 im Familien- zweig des Adda-kalla}

Bei Ibbi-Enlil ${ }^{55}$ (Generation 6), dem Sohn von Sîn-iqǐ̌am und vermuteten Enkelsohn von Adda-kalla, sind wir in der glücklichen Lage, seinen Erbteil direkt mit seinem eigenen Nachlass vergleichen zu können. Die Urkunde bezüglich seines Nachlasses (OECT 8,19$)$ trägt das Datum Rìm-Sîn II b/10/25, was dem Jahr Samsu-iluna 9 entspricht. ${ }^{56}$ Das Datum der älteren Urkunde mit dem Erbteil des Ibbi-Enlil ist nur zum Teil erhalten, s. MC 3, Nr. 26 iv 13-15 nach Foto Stone/Owen (1991) Pl. 4: ${ }^{13}$ [itin] e - n e - $\hat{g}$ a r 14 [m u sa-a]m-su-i-lu-na lugal ${ }^{15}[\ldots]{ }^{\top}{ }^{\top} \mathrm{NE}^{\text {?] }}$ (abgebro-

54 Die Angaben in Tabelle 4 beziehen sich jeweils auf die Summe aus Vorzugsanteil und Normalteil.

55 Stone/Owen $(1991,66)$ Nr. 29: 19 lasen [ib]-ni-den-líl. Mit Van De Mieroop (1991-1993, 125a zu Text 29) ist aber besser [i-b]i-den-líl zu lesen. Die vierte Zeile des Siegels fasst die Erben entsprechend zusammen als ibila 'i $i$-[bi]-[d][en-líl], s. Stone/Owen (1991) Pl. 59-60. 56 Zur Datierung von Rīm-Sîn II s. Stol (1976, 44-58), Rositani (2003, 16-24); vgl. auch Charpin (2004, 338f.). 
Tabelle 4: Die Erbteile der ältesten Erben in Generation 6 im Familienzweig des Nanna-meša und im Familienzweig des Adda-kalla

\begin{tabular}{|c|c|c|}
\hline $\begin{array}{l}\text { Geteilte } \\
\text { Besitztümer }\end{array}$ & $\begin{array}{l}\text { Erbteil des Mannum-mēšu-lișșur } \\
\text { aus dem Nachlass seines Großvaters } \\
\text { Nuska-amah, } \\
\text { MC 3, Nr. } 51 \text { (Si 10/03/-) }\end{array}$ & $\begin{array}{l}\text { Erbteil des Ibbi-Enlil aus dem Nach- } \\
\text { lass seines Vaters Sîn-iqišam, } \\
\text { MC 3, Nr. } 26 \text { (Si 6?/05/-) }\end{array}$ \\
\hline \multirow{4}{*}{$\begin{array}{l}\text { Pfründen: } \\
\text { nam-é-da-di (den-líl dnin-líl) } \\
\text { nam-ì-du }{ }_{8} \text { ká gal } \\
\text { nam-ì-du }{ }_{8} \text { ká gu-la }\end{array}$} & & \\
\hline & 99 Tage/Jahr & 99 Tage/Jahr ([18] + 81) \\
\hline & - & $1[2,5 ?]$ Tage/Jahr \\
\hline & 1[2(,5)?] Tage/Jahr & - \\
\hline nam-ì-du 8 ká é-gal maȟ & 1[2(,5)?] Tage/Jahr & - \\
\hline $\begin{array}{l}\text { nam-ì-du }{ }_{8} \text { é//ká ús-gíd(-gíd)-da } \\
\text { nam-ì-du }{ }_{8} \text { ká gal dnin-líl-lá }\end{array}$ & $\begin{array}{l}1\left[2(, 5)^{?}\right] \text { Tage/Jahr } \\
1\left[2(, 5)^{?}\right] \text { Tage/Jahr }\end{array}$ & 12,5 Tage/Jahr von beiden Pfründen \\
\hline nam-ì-du ${ }_{8}$ ká ĝeš-kîĝti & 12 Tage/Jahr & 12,5 Tage/Jahr \\
\hline 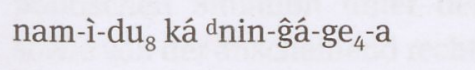 & 6 Tage/Jahr & 8 Tage/Jahr \\
\hline nam-ì-du ${ }_{8}$ é//ká du -kù(-ga) & 12,5 Tage/Jahr & {$[3,5]$ Tage/Jahr } \\
\hline 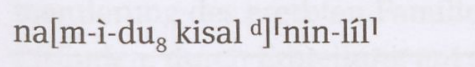 & 1,25 Tage/Jahr & - \\
\hline [nam-x x x (x) l]a-ma-sà-tum & 2,5 Tage/Jahr & - \\
\hline $\begin{array}{l}\text { nam-gudu }{ }_{4} \text { den-líl } u_{18} \text {-ru-mah- } \\
\text { an-ki }\end{array}$ & 22 Tage/Jahr & - \\
\hline nam-ì-du ${ }_{8}$ ká níĝ-ur ${ }_{5}-r a$ & 12 Tage/Jahr & - \\
\hline Wohnhaus & [1]2 gig $\hat{g}_{4}$ é dù-a & 44 gi $\hat{g}_{4}$ é dù-a \\
\hline Feld & - & $\begin{array}{l}93 \text { sar a-šà uz-za } \\
1 \text { iku a-šà (i }{ }_{7} \text {-)ĝìii(ki)-ta-bala-e }\end{array}$ \\
\hline zà-gu-la-Tisch & 1 & - \\
\hline
\end{tabular}

chen). Es ist vor dem Datum der Kaufurkunde MC 3, Nr. 28 ( $\mathrm{Si}$ 08/01/14) anzusetzen, in welcher Ibbi-Enlil seinen gesamten ererbten Hausanteil an Mannum-mēšu-lișșur verkaufte; und wahrscheinlich auch vor dem Datum der Heirats- und Adoptionsurkunde MC, Nr. 27 (Si 6/07/-), mit der Ibbi-Enlils jüngerer Bruder, Nannāja, seinen Erbteil an vier Adoptivsöhne übertrug. ${ }^{58}$ Möglicherweise bezeugen die Spuren der Datenformel das Jahr Samsuiluna 6: ${ }^{13}$ [itin] e-ne-ĝ ar ${ }^{14}$ [mu sa-a]m-su-i-lu-na lugal ${ }^{15}\left[\right.$ [... šù d] $u^{\text {?-Id }}$ è?l (abgebrochen). ${ }^{59}$

57 Siehe Neubearbeitung Anhang 1.

58 Siehe Textzitat Anm. 35.

59 Nach Horsnell (1999, Vol.2, 185-187) lautet der Jahresname Samsu-iluna 6 in seiner ausführlichsten Form: mu sa-am-su-i-luna lugal-e dutu dmarduk-e-ne-bi-da-ra níĝ-dím-dímma-bi al in-na-an-du $u_{11}-u s ̌(-a ̀ m)$ alan šùdu(-šùdu)-dè dlamma kù-si $i_{22}$ didli-bi-ta níĝ-si?-sá? ab?-di-di-dè
In diesem Fall lagen zwischen Erhalt des Erbteils und Übertragung an die Adoptivsöhne seitens des jüngeren Bruders Nannāja nur zwei Monate (Si 6?/05/- bzw. Si 6/07/-). Sein älterer Bruder Ibbi-Enlil behielt seinen Erbteil etwas länger: Bis zum Verkauf seines ererbten Hausteils an Mannum-mēšu-lișșur (Si 08/01/14) vergingen gut eineinhalb Jahre, bis zur Vererbung seines restlichen Erbteils an seine Söhne (RS II b/10/25 = Si 9) dreieinhalb Jahre; zwischen Verkauf und Vererbung war Ibbi-Enlil offenbar verstorben. Tabelle 5 bietet einen Überblick über Erbteil und Nachlass Ibbi-Enlils:

é-babbar igi dutu-šè é-saĝ-íl igi dmarduk-šè i-ni-inku, -ra ki-gub-ba-ne-ne mi-ni-in-ge-na; die kürzeste Form lautet: mu alan šùdu-dè. 
Tabelle 5: Erbteil und Nachlass des Ibbi-Enlil, Sohn von Sîn-iqišam

\begin{tabular}{lll}
\hline $\begin{array}{l}\text { Geteilte } \\
\text { Besitztümer }\end{array}$ & $\begin{array}{l}\text { Erbteil }^{60} \text { des Ibbi-Enlil aus dem } \\
\text { Nachlass seines Vaters Sîn-iqišam, }\end{array}$ & $\begin{array}{l}\text { Gesamter Nachlass des Ibbi-Enlil, } \\
\text { Sohn von Sîn-iqišam, }\end{array}$ \\
& MC 3, Nr.26 (Si 6?/05/-) & OECT 8, 19 (RS II 03/10/25 = Si 9)
\end{tabular}

\begin{tabular}{|c|c|c|}
\hline $\begin{array}{l}\text { Pfründen: } \\
\text { (nam-)é-da-di (den-líl dnin-líl-lá) }\end{array}$ & 99 Tage/Jahr & 70 Tage/Jahr \\
\hline nam-ì-du ${ }_{8}$ ká gal & 1[2,5?] Tage/Jahr & 12,5 Tage/Jahr \\
\hline $\begin{array}{l}\text { nam-ì-du }{ }_{8} \text { ká ús-gíd-gíd-da } \\
\text { ù ká gal dnin-líl-lá }\end{array}$ & 12,5 Tage/Jahr & $\begin{array}{l}\text { 12,5 Tage/Jahr (é ús-gíd-da kisal } \\
\text { é?-gal??-mah̆ ká dnin-líl-lá) }\end{array}$ \\
\hline nam-ì-du ${ }_{8}$ ká ĝeš-kị́̂-ti & 12,5 Tage/Jahr & 12,5 Tage/Jahr \\
\hline nam-ì-du $u_{8}$ ká dnin-ĝá-ge ${ }_{4}-a$ & 8 Tage/Jahr & 6 Tage/Jahr \\
\hline nam-ì-du ${ }_{8}$ ká du 6 -kù(-ga) & [3,5] Tage/Jahr & 12 Tage/Jahr \\
\hline Wohnhaus & 44 giğ $_{4}$ é dù-a & - \\
\hline Feld & 88 sar a-šà uz-za & - \\
\hline & 1 iku a-šà (i-) $\left.i_{7}\right)$ giriri(ki)-ta-bala-e & - \\
\hline
\end{tabular}

Festzustellen ist eine Verringerung des Wohnhauses. Wie gerade schon angesprochen, verkaufte Ibbi-Enlil sein gesamtes ererbtes Haus von $44 \mathrm{gi} \hat{\mathrm{g}}_{4}\left(26,4 \mathrm{~m}^{2}\right)$ für $1 / 3$ Mine Silber an Mannum-mēšu-lișșur, s. MC 3, Nr. 28. Was mit den zwei von Ibbi-Enlil ererbten Feldern geschah, wissen wir nicht. Vielleicht wurden sie ebenfalls verkauft, vielleicht wurden sie nicht in der Erbteilungsurkunde aufgeführt, weil seine Söhne sie in Erbengemeinschaft bewirtschafteten, vielleicht war ihre Teilung in einer separaten Erbteilungsurkunde dokumentiert - viele Möglichkeiten sind denkbar. Von den ererbten 148 Tagen pro Jahr an sechs verschiedenen Pfründenämtern vererbte Ibbi-Enlil 125,5 Tage weiter. Das ist ein Minus von 15 Prozent, ohne Berücksichtigung sicherlich bestehender Wertunterschiede zwischen den einzelnen Pfründen, über die wir leider wenig wissen.

Die Minderung des Familienvermögens wird besonders deutlich, wenn man z.B. den Erbteil von Ninurtamuštāl (Generation 7) mit dem Erbteil seines Urgroßvaters Adda-kalla (Generation 4) vergleicht: Erbte Adda-kalla Haus, Hof, Feld, 152,5 Tage von acht verschiedenen Pfründen und Mobilien, so verzeichnet die Urkunde über die Teilung des Nachlasses von Ibbi-Enlil für Ninurta-muštāl nur 48,5 Tage von drei verschiedenen Pfründen.

60 Die Angaben beziehen sich auf die Summe aus Vorzugsanteil und Normalteil des Ibbi-Enlil.
Die Pfründen von Ninurta-muštāl gingen zudem vollständig in den Besitz von Mannum-mēšu-lișșur über: Knapp ein Jahr nach der Teilung des Nachlasses von IbbiEnlil machte Mannum-mēšu-lișșur offenbar in einem Prozess Ansprüche gegen die Erben von Ibbi-Enlil geltend, weil er für ihren Vater Ausgaben in Form von 2 Kor (ca. 600 1) Gerste auf sich genommen hatte. ${ }^{61}$ Der Grund für diese Ausgaben geht aus der Urkunde leider nicht hervor. Handelte es sich um eine Art zinsloses Darlehen innerhalb der Familie? Die Formulierung: 2 še gur ù nîg $-g u_{7}$ $\mathrm{mu}$ 1-a-kam, „2 Kor Getreide und Speise in einem Jahr“, könnte darauf hinweisen, dass sich Ibbi-Enlil in einer Notsituation befand und das Getreide im unruhigen Jahr Samsu-iluna 9, in welchem der Usurpator Rìm-Sîn II in Nippur herrschte, zur Ernährung seiner Familie benötigte. ${ }^{62}$ Mannum-mēšu-lișșur konnte seine Ausgaben für Ibbi-Enlil mit einer Urkunde belegen. Die Erben des IbbiEnlil, Ninurta-muštāl, Namāršu-lūmur und Munawwirum, mussten ihn daraufhin für seine Ausgaben entschädigen und übereigneten ihm 30 Tage é - d a - d i -Pfründe für Enlil und Ninlil. Wahrscheinlich gab dafür jeder der drei Erben 10 Tage von seinem ererbten Anteil dieser Pfründe. Fünf Monate später kaufte Mannum-mēšu-lișșur dem Ninurta-

61 OECT 8, 11 (Si 10/09/03; s. Stone/Owen 1991, Nr. 31).

62 Van De Mieroop (1991-1993, 127b zu Text 31) vermutete, dass Mannum-mēšu-lișșur dem Ibbi-Enlil die 2 Kor Getreide als Unterhalt zahlte, weil dieser ihn adoptiert hatte. Für Kritik an dieser These s. Anm. 42. 
muštāl seine restlichen ererbten Pfründen $a b{ }^{63}$ Die Familie Ninurta-muštāls war offenbar in der Lage, erneut é-da-di-Pfründe zu erwerben, die Mannum-mēšu-lișșur nach weiteren zehn Monaten von Sohn und Frau Ninurtamuštāls, Inbi-iliı̌u und Lamassatum, erwarb. ${ }^{64}$ Anlass zu diesem letzten Kauf gab wahrscheinlich der Tod Ninurtamuštāls, der wie schon sein Vater Ibbi-Enlil nicht lange nach Erhalt seines Erbteils verstorben war.

Adda-kallas Urenkel Ninurta-muštāl veräußerte somit seinen gesamten Familienbesitz an Mannum-mēšulișșur. ${ }^{65}$ Die Erbteile seiner jüngeren Brüder, Namāršulūmur und Munawwirum, waren mit insgesamt 44,5 bzw. 32,5 Tagen/Jahr von drei bzw. zwei Pfründen nicht sehr groß. Dieser Befund weist auf eine Verarmung im Familienzweig des Adda-kalla in den Generationen 6-8. Begünstigt wurde sie möglicherweise von der unruhigen politischen Situation unter dem Usurpator Rìm-Sîn II sowie von der anscheinend recht kurzen Lebensdauer von Ibbi-Enlil und seinem Sohn Ninurta-muštāl, die eine Fragmentierung des ererbten Familienbesitzes, besonders der Pfründen, durch Erbteilung mit sich brachte.

\subsection{Die Entwicklung des Familienvermögens in den Generationen 6 und 7 im Familienzweig des Nanna-meša}

Mannum-mēšu-lișșur (Generation 7) scheint durch Käufe Nutzen aus der wirtschaftlich schwierigen Situation des Familienzweiges von Adda-kalla gezogen zu haben. Vielleicht war er aber auch nur bemüht, das Familieneigentum zusammenzuhalten, was den Angehörigen dieses Familienzweiges nicht möglich war. Vergleicht man seinen Erbteil mit dem Erbteil seines Urgroßvaters Nanna-meša (Generation 4), so sieht die Situation weit besser aus als im Familienzweig des Adda-kalla: Mannum-mēšu-lișșur erbte zwar nur $12 \mathrm{gi}_{4}\left(7,5 \mathrm{~m}^{2}\right)$ Haus $^{66}$, gut ein Siebtel der von Nanna-meša ererbten $80 \mathrm{~g} \mathrm{i}_{4}\left(48 \mathrm{~m}^{2}\right)$, und tauschte diese kleine Hausfläche gleich nach Erhalt gegen Pfründen seines Onkels und Miterben Munawwirum ein, ${ }^{67}$ doch

63 MC 3, Nr. 33 (Si 11/02/01): 20 Tage/Jahr nam-é-da-di den-líl dnin-líl, 12,5 Tage/Jahr ká ús-gíd-da kisal é-gal mah ká dnin-líl-lá und 6 Tage/Jahr ká dnin-ĝá-ge $e_{4}$-a für einen Kaufpreis von 3,5 Schekel Silber.

64 MC 3, Nr. 37 (Si 11/12/-): Anzahl Tage nicht erhalten; Kaufpreis 2 Schekel Silber.

65 Vielleicht mit Ausnahme der beiden Felder im Erbteil des IbbiEnlil (MC 3, Nr. 26 i 13-15. ii 7-9), deren Verbleib in den späteren Urkunden nicht dokumentiert ist.

66 Siehe Anhang 1, Cornell 23: 1. 12, mit Anmerkung dazu.

67 OECT 8, 16 (Si 10/03/23; s. Stone/Owen 1991, Nr. 30). erhielt er von den ererbten Pfründen seines Urgroßvaters immerhin noch gut die Hälfte.

Die Verminderung des ererbten Familienvermögens gegenüber den vorhergehenden Generationen konnte Mannum-mēšu-lișșur durch zahlreiche Käufe ausgleichen. Über drei Jahre hinweg (Si 11 bis Si 13) kaufte er Pfründen, oft solche, die er selbst in seinem Erbteil erhalten hatte. ${ }^{68}$ Außerdem erwarb er in einem vergleichsweise kurzen Zeitraum von zweieinhalb Monaten ( $\mathrm{Si}$ 12/03/15 bis Si 12/06/01) 3 i ku (1,08 ha) Stoppelfeld und 2 s a r $\left(72 \mathrm{~m}^{2}\right)$ Haus möglicherweise für den Bedarf seiner eigenen Familie zum Zeitpunkt der Familiengründung. ${ }^{69}$ Damit ist Mannum-mēšu-lișșurs Besitz an Feld und Haus durchaus mit dem seines Urgroßvaters Nanna-meša vergleichbar, der insgesamt 3 iku 60 sar (1,3 ha) Feldfläche, $1 \frac{1 / 3}{3}$ sar $\left(48 \mathrm{~m}^{2}\right)$ Wohnhaus und 7 sar $\left(252 \mathrm{~m}^{2}\right)$ unbebautes Hausgrundstück erbte.

Für seine Käufe in den Jahren Samsu-iluna 11-13 gab Mannum-mēšu-lișșur insgesamt rund eine Mine Silber aus. $^{70}$ Es stellt sich die Frage, woher er dieses Silber nahm? Dass er seinen Reichtum mit Stone (1991, 29-33) der Adoption durch Nannāja verdankte, ist zu bezweifeln (s. Abschnitt 3.4): Mannum-mēšu-lișșur erhielt dadurch zwar einen Anspruch auf einen Erbteil aus dem Nachlass seines Adoptivvaters, aber kein direkt verfügbares Kapital. ${ }^{71}$ Stattdessen stand Mannum-mēšu-lișșur für seine Kauftätigkeit der Erbteil seines verstorbenen Vaters Awìlija aus dem Nachlass seines Großvaters Nuska-amah zur Verfügung, den er im Jahr Samsu-iluna 10 erhielt.

Der Erbteil bestand im Wesentlichen aus Pfründenämtern. Diese waren gerade in der Regierungszeit Samsuilunas ein gefragtes Kaufgut. Wie hoch die damit verbun-

68 MC 3, Nr. 33 (Si 11/02/01); OECT 8, 6 (Si 11/09/16, s. Stone/Owen 1991, Nr. 34); MC 3, Nr. 35 (Si 11/10/2); Nr. 36 (Si 11/11/08; Käufer wahrscheinlich Mannum-mēšu-lișșur, s. Anm. 2); Nr. 37 (Si 11/12/-); OECT 8, 5 (Si 12/03/-, s. Stone/Owen 1991, Nr. 39); OECT 8, 9 (Si 12/04/21, s. Stone/Owen 1991, Nr. 41); TIM 4, 54 (Si 12/06/[...], s. Stone/Owen 1991, Nr. 43); MC 3, Nr. 44 (Si 12/10/20); OECT 8, 10 (Si 12/10/24, s. Stone/Owen 1991, Nr. 45); MC 3, Nr. 47 (Datum abgebr.); Nr. 48 (Datum abgebr.); OECT 8, 8 (Si 13/04/05, s. Stone/Owen 1991, Nr. 49); OECT 8, 7 (Si 13/12/02, s. Stone/Owen 1991, Nr. 50).

69 Feldkauf: 3 iku in OECT 8, 2 (Si 12/03/15; s. Stone/Owen 1991, Nr. 38). Hauskäufe: 1 1/3 s s r in BE 6/2, 38 (Si 12/04/06; s. Stone/Owen 1991, Nr. 40; das Hausgrundstück wird 17 Jahre später anscheinend von der Familie der Verkäufer wieder zurückgekauft, s. BE 6/2, 64); 2/3 s a r in OECT 8, 1 (Si 12/06/01; s. Stone/Owen 1991, Nr. 42).

70 Die Summe aller erhaltenen Preiszahlungen beträgt 47,75 Schekel Silber (ca. 400 g); hinzukommen vier Kaufurkunden, in denen die Kaufpreise nicht erhalten sind: MC 3, Nr. 35, Nr. 36, Nr. 44; OECT 8, 8 (s. Stone/Owen 1991, Nr.49). In der ersten dieser drei Urkunden ist auch der Name des Käufers nicht erhalten, sehr wahrscheinlich handelt es sich aber um Mannum-mēšu-lișșur, s. Anm. 2.

71 Siehe MC 3, Nr. 27; Textzitat s. Anm. 35. 
denen Einkünfte in altbabylonischer Zeit waren, wissen wir leider nicht. Doch selbst wenn sie nur gering gewesen sein sollten, waren Pfründeneinkünfte vermutlich ansehnlich, wenn sie sich über einen längeren Zeitraum hinweg ansammelten. ${ }^{72}$ Das trifft bei Mannum-mēšu-lișșur zu. Die von Nuska-amah ererbten Pfründen wurden mindestens 22 Jahre lang (Ha 31 bis Si 10) in Erbengemeinschaft verwaltet und die Einkünfte daraus vermutlich geteilt, zunächst zwischen Awilija und Munawwirum und nach Awilijas Tod zwischen Mannum-mēšu-lișșur und Munawwirum. ${ }^{73}$ Wahrscheinlich waren es diese Einkünfte, die Mannummēšu-lișșur das notwendige Kapital für seine Käufe verschafften und ihn außerdem in die Lage versetzten, einen Kauf bereits vor dem Erhalt seines Erbteils zu tätigen. ${ }^{74}$

\subsection{Das Familienvermögen in der Generation 8 im Familienzweig des Nanna-meša}

Die jüngste Urkunde, die Auskunft über Vermögen der Familie des Mannum-mēšu-lișșur gibt, ist BE 6/2, 64 (Si 29/02/20). Sie dokumentiert den Rückkauf von 1 1/3 sar $\left(48 \mathrm{~m}^{2}\right)$ unbebauten Hausgrundstücks (é kislaḩ). Dieses Grundstück hatte Mannum-mēšu-lișșur von den Söhnen des Ea-iddinam gekauft. Wahrscheinlich liegt die Kaufurkunde in BE 6/2, 38 ( $\mathrm{Si} 12 / 04 / 06)$ vor. Dort wird das seitens Mannum-mēšu-lișșur von den Söhnen des Ea-iddinam gekaufte, $48 \mathrm{~m}^{2}$ große Grundstück zwar als é d ù - a , „Wohnhaus“, bezeichnet, doch besteht die Möglichkeit, dass das auf dem Grundstück errichtete Haus in den dazwischenliegenden 17 Jahren verfallen und das Grundstück beim Rückkauf daher nicht mehr bebaut war. ${ }^{75}$

72 So auch Stone $(1977,284)$ : „Temple office ownership, representing as it did participation in one of the large concerns of the city, the temple, yielded a modest, but relatively secure income to its holder. (...) ownership of sufficient temple office property provided a secure income which allowed the accumulation of other property (...)“

73 Nuska-amah war spätestens zu Beginn des Jahres Hammurapi 31 verstorben, s. OECT 8, 4 (Ha 31/05/-; s. Stone/Owen 1991, Nr. 25); Mannum-mēšu-lișșurs Vater Awilija vermutlich spätestens im Jahr Samsu-iluna 6, s. MC 3, Nr.27 (Si 6/07/-). Siehe dazu bereits Abschnitte 3.3 und 4.1 .

74 Mannum-mēšu-lișșur kaufte zu Beginn des Jahres Samsu-iluna 8 das von Ibbi-Enlil, Sohn des Sîn-iqī̌am, ererbte Wohnhaus von 44 gi $\hat{\mathrm{g}}_{4}\left(26,4 \mathrm{~m}^{2}\right)$ für $1 / 3$ Mine (= $166 \mathrm{~g}$ ) Silber, s. MC 3, Nr. 28.

75 Allerdings ist der Preis für den Rückkauf des é kislah mit 6,5 Schekeln Silber (BE 6/2, 64: 14; Si 29/02/20; s. Stone/Owen 1991, Nr. 52) deutlich höher als der Preis von ${ }^{1} 3^{1}(+)[($ Bruchteil? $)]$ Schekeln Silber für den Kauf des é d ù - a (BE 6/2, 38: 13; Si 12/04/06; s. Stone/ Owen 1991, Nr. 40). Eine Lesung der beschädigten Zahl in letzterem Beleg als ${ }^{\mathrm{F}} 6 \mathrm{~T}$ ist nach Kollation unwahrscheinlich.
Im Jahr Samsu-iluna 29 nun kauft Ninurta-rā'imzērim, Sohn von Ninurta-manšum, ein Nachbar des Grundstücks, dieses als Teil des Besitzes seiner Familie zurück (é ad-da-na in-du $u_{8}$ ). Verkäufer ist nicht Mannum-mēšu-lișșur selbst, sondern seine nächsten Anverwandten: sein Bruder Ninurta-muballiț, Sohn von Awīlija, Iddin-Ištar, Sohn von Mannum-mēšu-lișșur, und Iddin-Ištars Mutter Narubtum, die Frau von Mannummēšu-lișșur. Das bedeutet, dass Mannum-mēšu-lișșur zu diesem Zeitpunkt verstorben war. Vielleicht hatte sein Tod die Voraussetzung für den Rückkauf geschaffen und sich kurz zuvor ereignet.

Der letzte Beleg, der Mannum-mēšu-lișșur am Leben zeigt, ist die gut 15 Jahre ältere Kaufurkunde OECT 8, 7 (Si 13/12/02), in der Mannum-mēšu-lișșur als Käufer auftritt. Unter der Annahme, dass uns das Archiv des Mannummēšu-lișșur in seinen wesentlichen Teilen bekannt ist, würde das bedeuten, dass Mannum-mēšu-lișșur in den drei Jahren nach Erhalt seines Erbteils massiv Pfründen kaufte, die restlichen 15 Jahre seines Lebens aber keinerlei Kauftätigkeit mehr an den Tag legte. Vielleicht hatte er mit seinen Käufen für seinen Lebensunterhalt ausgesorgt und konnte anschließend vom Gewinn aus seinen Pfründen leben?

Die Verkäufer an Mannum-mēšu-lișșurs statt sind ganz offensichtlich seine nächsten Anverwandten und damit die Personen, die Ansprüche auf seinen Nachlass geltend machen konnten. ${ }^{76}$ Der Umstand, dass unter ihnen nur ein Sohn Mannum-mēšu-lișșurs genannt ist, macht es wahrscheinlich, dass Mannum-mēšu-lișșur nur diesen einen Sohn Iddin-Ištar hatte. Vermutlich übernahm er als einziger Erbe den gesamten Nachlass seines Vaters, nachdem diesem die Mitgift seiner Mutter und etwaige Schenkungen an sie entnommen worden waren sowie mögliche Besitztümer, auf die Ninurta-muballit vielleicht infolge einer Erbengemeinschaft mit Mannummēšu-lișșur Besitzansprüche hatte. Mit dem Nachlass übernahm Iddin-Ištar vermutlich auch alle Urkunden, welche Erwerbungen und Besitzansprüche des Mannummēšu-lișșur dokumentierten.

Was weiter mit Iddin-Ištar und dem Nachlass seines Vaters geschah, wissen wir leider nicht, da keine späteren Urkunden bekannt sind, die sich dem Archiv des Mannum-mēšu-lișșur zuordnen ließen, und der Urkundenstrom aus dem altbabylonischen Nippur wenig später ganz versiegt. ${ }^{77}$

76 Für Überlegungen zu den rechtlichen Hintergründen des gemeinsamen Auftretens von Ehefrau bzw. Bruder eines Verstorbenen und dessen Erben als Verkäufer, speziell auch in Hinsicht auf BE 6/2, 64, s. Meinhold (2014, 23-27). 77 Siehe Charpin (2004, $360 \mathrm{f}$.). 


\section{Zusammenfassung}

Das Vermögen der Familie des Mannum-mēšu-lișșur blieb bis zur fünften Generation weitgehend konstant, vermindert nur durch Erbteilungen. Solcher Verminderung entgegenwirkend sind durch alle Generationen hinweg Tendenzen zu beobachten, das Erbe zusammenzuhalten: Nuska-amah (Generation 5), Sohn von Nanna-meša, scheint die ererbten Pfründen seines Vaters ungeteilt übernommen zu haben, vermutlich gegen Ausgleichszahlungen an seine Brüder; Nannāja und Ibbi-Enlil (Generation 6) teilten nicht nur den Nachlass ihres Vaters Sîn-iqišam, sondern zugleich auch den Erbteil ihres Onkels Ilī-āpilī, den dieser ihnen vermacht hatte; Mannum-mēšu-lișșur (Generation 7) erhielt den vollständigen Erbteil seines verstorbenen Vaters Awilija und musste ihn nicht mit seinen Brüdern teilen.

$\mathrm{Ab}$ der sechsten Generation entwickelte sich das Familienvermögen unterschiedlich: Während der Familienzweig des Adda-kalla verarmte und nach zwei frühen Toden der Familienoberhäupter Ibbi-Enlil und Ninurtamuštāl (Generationen 6-7) sogar gezwungen war, das gesamte ererbte Familienvermögen zu veräußern, war Mannum-mēšu-lișșur (Generation 7) im Familienzweig des Nanna-meša in der Lage, dieses und weiteres Vermögen aufzukaufen. Das Silber für seine Käufe bezog er vermutlich aus den Einkünften ererbter Pfründen, von denen er als Teilhaber einer Erbengemeinschaft bereits vor der Erbteilung im Jahr Samsu-iluna 10 profitierte.

Die gegensätzlichen Entwicklungen des Vermögens der beiden Familienzweige vollzogen sich im ersten Drittel der Regierungszeit Samsu-ilunas, welches durch eine wirtschaftliche, politische und militärische Krise gekennzeichnet war. ${ }^{78}$ Diese Krise brachte offensichtlich wirtschaftliche Gewinner und Verlierer selbst innerhalb einer Familie hervor.

Nicht aufrechterhalten werden kann die These Stone's, dass die Adoptionen in der Familie des Mannummēšu-lișșur in erster Linie der Übertragung von Vermögen gedient hätten und rein ökonomisch motiviert gewesen seien. ${ }^{79}$ Zwar spielten Vermögensübertragungen in Form von Erbeinsetzung, Unterhaltszahlung oder Einmalzahlung bei allen Adoptionen eine Rolle, daneben aber auch soziale Faktoren, wie der Bedarf an einem Erben und Versorger im Alter mangels eigener leiblicher Söhne

$78 \mathrm{Zu}$ dieser Krise in den Jahren Si 9-13 s. Stone (1977), Bobrova/ Koshurnikov (1989), Charpin (2004, 335-346).

79 Für eine kritische Bewertung der Differenzierung Stone's von „familial adoptions“ und „economic adoptions“ s. auch Wunsch (2003-2004, 185ff.) und Obermark (1992, Part I, 12-26).
(Adoption des Lu-Bau durch Ur-Lumma) oder die Eingliederung von Kindern aus einer früheren Ehe in eine durch erneute Eheschließung begründete Familie (Adoption von Mannum-mēšu-lișșur, Ninurta-muballiț, Sîn-māgir und Ina-Ekur-rabi durch Nannāja). Einzig die Adoption des Ninurta-muballit durch die Eheleute Ilī-ippalsam und Elmēšum könnte vorrangig ökonomisch motiviert gewesen sein, insbesondere wenn Ninurta-muballit dadurch in ein zeitgleich bestehendes zweites Adoptionsverhältnis eingetreten wäre. Mannum-mēšu-lișșur hingegen verdankte seine Kaufkraft und seine soziale Stellung nicht der Adoption durch Nannāja, sondern seinem ererbten Vermögen in Form zahlreicher Pfründen.

\section{Literatur}

Bobrova, L. V./S. G. Koshurnikov (1989): On some new works in the social history of the Old Babylonian period, AoF 16, $51-60$

Charpin, D. (1980): Archives familiales et propriété privée en Babylonie ancienne: Étude des documents de «Tell Sifr». Paris

Charpin, D. (1986): Transmission des titres de propriété et constitution des archives privées en Babylonie ancienne, in: K. R. Veenhof (Hg.), Cuneiform Archives and Libraries. CRRAI 30. PIHANS 57. Leiden, 121-140

Charpin, D. (1994): Rezension zu Stone/Owen 1991, JAOS 114, 94-96

Charpin, D. (2004): Histoire politique du Proche-Orient amorrite (2002-1595), in: D. Charpin/D. O. Edzard/M. Stol, Mesopotamien. Die altbabylonische Zeit. Annäherungen 4. OBO 160/4. Friboug/Göttingen, 23-480

Dijk, J. van (1967): Texts in the Iraq Museum. Old Babylonian contracts and juridical texts. Volume IV. Wiesbaden

Horsnell, M. J. A. (1999): The year names of the First Dynasty of Babylon. Volume 1-2. Hamilton, Ontario

Hunter, G. R. (1930): The Sayce and H. Weld Collection in the Ashmolean Museum. Sumerian contracts from Nippur. OECT 8. London

Janssen, C./H. Gasche/M. Tanret (1994): Du chantier à la tablette. Ur-Utu et l'histoire de sa maison à Sippar-Amnānum, in: H. Gasche et al. (Hg.), Cinquante-deux réflexions sur le ProcheOrient ancien offertes en hommage à Léon De Meyer. MHEO 2, Leuven

Klíma, J. (1940): Untersuchungen zum altbabylonischen Erbrecht. Prag

Kraus, F. R. (1951): Nippur und Isin nach altbabylonischen Rechtsurkunden. JCS 3

Lafont, B. (1992): Quelques nouvelles tablettes dans les collections américaines, RA 86, 97-111

Landsberger, B. (1937): Die Serie ana ittišu. MSL 1. Rom

Meinhold, W. (2014): Erbrecht nach altbabylonischen Kauf- und Tauschurkunden aus Nippur, ZAR 20, 11-32

Mitchell, J. R. (1991): Prosopographic index to the Mannummešu-lișșur archive, in: Stone/Owen 1991, 95-124 
Obermark, P. R. (1992): Adoption in the Old Babylonian period. Part I-II. Ph.D. Dissertation Hebrew Union College Ohio. University Microfilms International. Ann Arbor, Michigan

Obermark, P. R. (1993-1994): Rezension zu Stone/Owen 1991, AfO 40/41, 106-109

O'Callaghan, R. T. (1954): A new inheritance contract from Nippur, JCS 8, 137-143

Oelsner, J. (1993): Rezension zu Stone/Owen 1991, OLZ 88, 500-504

Otto, E. (1995): Rezension zu Stone/Owen 1991, ZA 85, 163-166

Pedersén, 0. (1998): Archives and libraries in the ancient Near East 1500-300 B. C. Bethesda

Poebel, A. (1909): Babylonian legal and business documents from the time of the First Dynasty of Babylon chiefly from Nippur. BE 6/2. Philadelphia

Rositani, A. (2003): Rìm-Anum texts in the British Museum. Nisaba 4. Rom
Stol, M. (1976): Studies in Old Babylonian history. PIHANS 40. Istanbul

Stone, E. C. (1977): Economic crisis and social upheaval in Old Babylonian Nippur, in: L. D. Levine/T. Cuyler Young (Hg.), Mountains and lowlands. Essays in the archaeology of greater Mesopotamia. BiMes 7. Malibu, 267-289

Stone, E. C. (1991): Adoption in Old Babylonian Nippur, in: Stone/ Owen 1991, 1-33

Stone, E. C./ D. I. Owen (1991): Adoption in Old Babylonian Nippur and the archive of Mannum-mēšu-lișșur. MC 3. Winona Lake

Prang (1976): Das Archiv des Imgûa, ZA 66, 1-44

Van De Mieroop, M. (1991-1993): Rezension zu Stone/Owen 1991, JCS 43-45, 124-130

Westbrook, R. (1988): Old Babylonian marriage law. AfO Beiheft 23. Horn

Wunsch, C. (2003-2004): Findelkinder und Adoption nach neubabyIonischen Quellen, AfO 50, 174-244

\section{Anhang 1:}

Cornell 23: Neubearbeitung der Erbteilungsurkunde mit Erbteil für Mannum-mēšu-lișșur

(Fotos: Stone/Owen, Pl. 27-28; http://cdli.ucla.edu/dl/photo/P270657.jpg; Kopie: MC 3, Nr. 51; CUSAS 15, Nr. 23; ältere Bearbeitung: Stone/Owen (1991, 87-89), Nr. 51; CUSAS 15, Nr. 23)

\section{Umschrift:}

Vs. $1 \quad 2^{\star}$ gi $\hat{g}_{4}$ é dù-a da é $i[m$-gur-dnin-urta $]$

2 nam-ì-dus ká gu-la mu-a u[ ${ }_{4} 2 \quad(1 / 2)$ ?- kam]

3 nam-ì-du $u_{8}$ ká é-gal mah mu-a $\left[\begin{array}{lll}u_{4} & 2(1 / 2) ?-k a m\end{array}\right]$

4 nam-ì-dus é* ús-gíd-da mu-a [u $u_{4} 2(1 / 2)$ ?-kam $]$

5 nam-ì-d $u_{8}$ ká gal dnin-líl-lá mu- $\mathrm{a}^{1}\left[\mathrm{u}_{4} 2(1 / 2)\right.$ ?-kam $]$

6 nam-ì-d $u_{8}$ ká $a^{\star}$ geš-kíĝ-ti mu-a $u_{4} 2-k a m$

7 nam-ì-du $u_{8}$ ká dnin-ĝá-ge $e_{4}-a \quad u_{4} \quad 1-k a m$

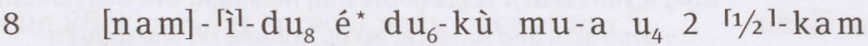

9 [nam-é]- $\mathrm{d}^{\mathrm{l}} \mathrm{l}-\mathrm{di}$ den-líl dnin-líl mu-a $\mathrm{u}_{4}$ 18-kam

10 [nam]-Igudu ${ }_{4}^{1}$ den-líl $u_{18}$-ru-mah-an-ki mu-a $u_{4}$ 4-kam

11 [1 ĝ̀šba]nsur zà gu-la síb-ta nam-ses-gal

$12\left[\begin{array}{ll}10 & \text { gi } \\ \hat{g}_{4}\end{array}\right]$ 'él-dù-a da é im-gur-dnin-urta

13 [nam-ì-d] $\mathrm{u}_{8}$ ká gu-la mu-a $\mathrm{u}_{4}$ 10-kam

14 [nam-ì-du $]_{8} 0^{*}$ é-Igall-mah mu-a $u_{4} 10-\mathrm{kam}$

$15\left[\right.$ [nam-ì-du $u_{8}$ 'él ús-gíd-da mu-a $u_{4} 10-k a m$

16 [nam-ì-du $u_{8}$ ká gal d]'nin'-líl-lá mu-a $u_{4}$ 10-kam

17 [nam-ì-du $u_{8}$ ká ĝeš-kíg]-ti mu-a $u_{4} 10-k a m$

18 [nam-ì-du $u_{8}$ ká dnin-ĝg]á- $\mathrm{ge} \mathrm{e}_{4}{ }^{1}-\mathrm{a}_{4} \quad \mathrm{u}_{4}$ 5-kam

19 [nam-ì-du $u_{8}$ é $\left.d u_{6}\right]-k u ̀ ~ m u-a ~ u_{4} 10-k a m$

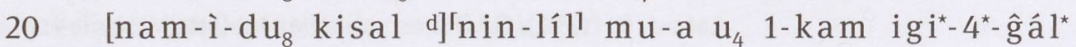

21 [nam-x x x (x) l] $a^{-}{ }^{-} m a^{-}$-sà-tum mu-a $u_{4} 2 \quad 1 / 2-k a m$

22 [nam-é-da-d]i den-líl dnin-líl-ke

23 [mu-a iti] ${ }^{2} 2-\mathrm{kam}^{\top} \mathrm{u}_{4}$ 21-kam

u.Rd. 24 「nam-gudu ${ }_{4}{ }^{\top}$ den-líl $u_{18}$-ru-mah-an-ki

$25 \mathrm{mu}$-àm $\mathrm{u}_{4} 18-\mathrm{kam}$ 
Rs. 26 nam-ì-du $u_{8}$ ká níĝ-ur-ra mu-a $u_{4}$ 12-kam

27 [h] a-la-ba ma-an-nu-um-me-šu-li-șur

$28 \quad[10 \mathrm{gig}]_{4}$ é dù-a da é ses-a-ni

29 [nam-ì-d] $u_{8}$ ká gu-la mu-a $u_{4}$ 10-kam

30 [nam-ì-du $]_{8} 0^{*}$ é-gal mah mu-a ${ }^{\top} \mathrm{u}_{4} \quad 10^{1}-\mathrm{kam}$

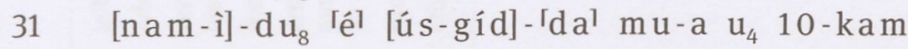

32 [nam-î]-du $u_{8}$ klá gal dnin-líll-lá mu-a u $u_{4}$ 10-kam

33 [nam]- $\Gamma_{i}-d u_{8}$ kál [ĝeš-kíĝ-t]i mu-a $u_{4}$ 10-kam

34 [na]m-ì- $\mathrm{d}_{\mathrm{d}} \mathrm{u}_{8}$ ká dnin-ĝá-ge $\mathrm{e}_{4}{ }^{\mathrm{l}}-\mathrm{a} \quad \mathrm{u}_{4}$ 5-kam

35 [na $] \mathrm{m}-\grave{i}-\mathrm{Idu}_{8}$ él $\mathrm{du}_{6}$-kù mu-a $\mathrm{u}_{4}$ 10-kam

36 [na]m-gudu $u_{4}\left[\text { den-líl }^{\mathrm{u}}\right]_{18}$-ru mah an-ki mu-a $\mathrm{u}_{4}$ 18-kam

37 na[m-ì-du kisal] [dnin-líl mu-a $u_{4} 1^{\star}$ igi-4*ấál

$38 \mathrm{na}[\mathrm{m}-\mathrm{x} \times \mathrm{x} \quad(\mathrm{x}) l a-m] a-s \grave{a}-t u\left[m \quad \mathrm{mu}-\mathrm{a} \quad \mathrm{u}_{4}\right] \quad 2 \quad \frac{1 / 2-\mathrm{kam}}{2}$

39 nam-[é-da-di den-líl d]'nin'-1[íl]

$40 \mathrm{~m}\left[\mathrm{u}^{*}-\mathrm{a}\right.$ iti $\left.2(-\mathrm{kam})\right] \mathrm{u}_{4}$ 21-kam

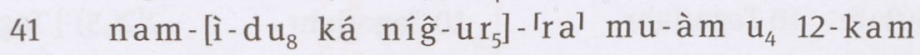

42 h้a-[la-ba mu-na]-wi-ru-um

43 ibila Idl[nuska-á]-mahn-ke $e_{4}-n e$

44 še-ga-ne-ne- Ita' ĝeš-šub-ba- Ita in-bal-eř

45 igi lú-den-líl-[lá agrig dumu é-lú-ti]

46 igi nu-ra-tum [gudu ${ }_{4}$ dnin-líl-lá (dumu lú-dnin-urta)]

47 igi ma-an-nu-um-ma-hir-šu d [u mu im-gur-dnin-urta]

48 igi dnuska-ni-šu [dub-sar]

o.Rd. 49 igi a-wi-li-ia b[ur-gul]

50 iti šeg ${ }_{12}$-a mu sa-am-su-[i ${ }^{-}$-[lu-na lugal]

51 usu mah ${ }^{\star} d^{\star} m[\operatorname{arduk}(-k a-t a)]$

Siegelabrollung:

1 ma-an-nu-um-me-šu-li-[șur]

2 dumu a-wi-li-i $[a]$

$3 \quad m u-n a-{ }^{\Gamma} w i-r u-u m{ }^{\top}$

4 dumu Idnuska-ál-[mah]

\section{Anmerkungen:}

$1210 \mathrm{gi} \hat{\mathrm{g}}_{4}$ ist ergänzt nach OECT 8, 16: 1-3 (Si 10/2/23), s. Stone/Owen $(1991,67)$ Nr. 30. Dieser Urkunde zufolge tauscht Mannum-mēšu-lișșur „12 gi $\hat{\mathrm{g}}_{4}$ Wohnhaus, seinen Normalteil und Vorzugsanteil, Erbteil des Mannum-mēšulișșur“, gegen drei Pfründen seines Onkels und Miterben Munawwirum. Mannum-mēšulișșurs Vorzugsanteil umfasst $2 \mathrm{~g} \hat{\mathrm{g}}_{4}$ (s. Cornell 23: 1), entsprechend muss der Normalteil 10 gi $\hat{\mathrm{g}}_{4}$ betragen.

20, 37 nam-ì-d $u_{8}$ kisal dnin-líl ist ergänzt nach OECT 8, 16: 8 (Si 10/2/23), wo gegen Stone/ Owen $(1991,67)$ Nr. 30 statt ká deutlich kisal zu lesen ist.
In den Tausch OECT 8, 16: 8 (Si 10/2/23) bringt Munawwirum für die ererbten $12 \mathrm{gi}_{4}$ Wohnhaus des Mannum-mēšu-lișșur drei Pfründen ein, nämlich 10,5 Tage/Jahr namì-du ${ }_{8}$ ká gu-la, 5 Tage/Jahr nam-ì-du ká dnin-ĝá-ge ${ }_{4}$-a und 1,5 Tage/Jahr namì- $d u_{8}$ kisal dnin-lîl-lá. Auch wenn in der Tauschurkunde nicht ausdrücklich vermerkt, handelt es sich dabei wohl um die gemäß Cornell 23: 29, 34 und 37 ererbten Pfründen des Munawwirum, z. T. um Bruchteile von Tagen vermehrt.

$2810 \mathrm{gi} \hat{\mathrm{g}}_{4}$ am Anfang der Zeile ergänzt gemäß Z. 12; s. Anmerkung zu dieser Zeile. 
45-49 Die Namen der Zeugen sind ergänzt nach der Tauschurkunde OECT 8, 16 (Si 10/2/23), welche dieselben Zeugen aufweist und vermutlich unmittelbar nach der Erbteilung ausgestellt wurde. Lies in OECT 8, 16: 18 mit den Fotos
(Stone/Owen 1991, Pl. 53; http://www.cdli. ucla.edu/dl/photo/P283643.jpg) nu-ra-tum, gegen Kopie und Stone/Owen $(1991,67)$ Nr. 30 nicht nu-ra-bi. Der Vatersname des Nūrātum ist ergänzt nach SAOC 44, 79: 7-8.

Tabellarische Übersetzung von Z.1-42:

\begin{tabular}{|c|c|c|c|c|}
\hline $\begin{array}{l}\text { Geteilte } \\
\text { Besitztümer }\end{array}$ & $\begin{array}{l}\text { Vorzugsanteil } \\
\text { des Mannum- } \\
\text { mēšu-lișșur }\end{array}$ & $\begin{array}{l}\text { Erbteil des } \\
\text { Mannum-mēšu- } \\
\text { lișșur }\end{array}$ & $\begin{array}{l}\text { Erbteil des } \\
\text { Munawwirum }\end{array}$ & $\begin{array}{l}\text { Gesamtes } \\
\text { Vermögen }\end{array}$ \\
\hline $\begin{array}{l}\text { Wohnhaus } \\
\text { Nachbar: }\end{array}$ & $\begin{array}{l}2 \mathrm{gig}_{4} \\
\text { I[mgur-Ninurta] }\end{array}$ & $\begin{array}{l}{\left[10 \text { gi }_{4}\right]} \\
\text { Imgur-Ninurta }\end{array}$ & $\begin{array}{l}{[10 \text { gî̂g }]_{4}} \\
\text { sein Bruder }\end{array}$ & [2]2 $\operatorname{gig}_{4}$ \\
\hline $\begin{array}{l}\text { Pfründen: } \\
\text { nam-ì-du } 8 \text { ká gu-la }\end{array}$ & {$\left[2(, 5)^{?}\right]$ Tage/Jahr } & 10 Tage/Jahr & 10 Tage/Jahr & 2[2(,5)?] Tage/Jahr \\
\hline nam-ì-du 8 ká é-gal maḩ & {$\left[2(, 5)^{?}\right]$ Tage/Jahr } & 10 Tage/Jahr & 10 Tage/Jahr & 2[2(,5)?] Tage/Jahr \\
\hline nam-ì-du $8 e^{\star} e^{\star} u ́ s-g i ́ d-d a$ & {$\left[2(, 5)^{?}\right]$ Tage/Jahr } & 10 Tage/Jahr & 10 Tage/Jahr & $2\left[2(, 5)^{?}\right]$ Tage/Jahr \\
\hline nam-ì-du ${ }_{8}$ ká gal dnin-líl-lá & {$\left[2(, 5)^{?}\right]$ Tage/Jahr } & 10 Tage/Jahr & 10 Tage/Jahr & $2\left[2(, 5)^{?}\right]$ Tage/Jahr \\
\hline nam-ì-du ${ }_{8}$ ká $a^{\star}$ geš-kí̂g-ti & 2 Tage/Jahr & 10 Tage/Jahr & 10 Tage/Jahr & 22 Tage/Jahr \\
\hline nam-ì-du ${ }_{8}$ ká dnin-ĝá-ge ${ }_{4}-a$ & $1 \mathrm{Tag} / \mathrm{Jahr}$ & 5 Tage/Jahr & 5 Tage/Jahr & 11 Tage/Jahr \\
\hline nam-ì-du 8 é du -kù $^{-}$ & 2,5 Tage/Jahr & 10 Tage/Jahr & 10 Tage/Jahr & 22,5 Tage/Jahr \\
\hline na[m-ì-du ${ }_{8}$ kisal] ${ }^{[d}$ nin-líl' & - & 1,25 Tage/Jahr & 1,25 Tage/Jahr & 2,5 Tage/Jahr \\
\hline $\mathrm{na}[\mathrm{m}-\mathrm{x} \times \mathrm{x}(\mathrm{x}) l] a-{ }^{-} m a^{1}$-sà-tum & - & 2,5 Tage/Jahr & 2,5 Tage/Jahr & 5 Tage/Jahr \\
\hline nam-[é]-「da'-di den-líl dnin-líl & 18 Tage/Jahr & 81 Tage/Jahr & {$[60]+21$ Tage/Jahr } & 180 Tage/Jahr \\
\hline $\begin{array}{l}\text { nam-gudu }{ }_{4} \text { den-líl u } \text { }_{18} \text {-ru-mah-an- } \\
\text { ki }\end{array}$ & 4 Tage/Jahr & 18 Tage/Jahr & 18 Tage/Jahr & 40 Tage/Jahr \\
\hline nam-ì-du 8 ká níĝ-ur ${ }_{5}$-ra & - & 12 Tage/Jahr & 12 Tage/Jahr & 24 Tage/Jahr \\
\hline zà-gu-la-Tisch & 1 & - & - & 1 \\
\hline
\end{tabular}

Übersetzung von Z. $43 \mathrm{ff}$.:

43 (Das) haben die Erben von [Nuska-a]mah

44 in gegenseitigem Einverständnis mit dem Los geteilt.

45 Vor Lu-Enlil[a, dem Verwalter, dem Sohn von Eluti],

46 vor Nūrātum, [dem gudu ${ }_{4}$-Priester der Ninlil(, dem Sohn von Lu-Ninurta)],

47 vor Mannum-māhniršu, dem S[ohn von Imgur-Ninurta],

48 vor Nuska-nīšu, [dem Schreiber],

o.Rd. 49 vor Awīlija, dem Sie[gelschneider].

50 Dritter Monat, Jahr: ,Samsu-i[luna, der König],

51 hat mit der hervorragenden Kraft M[arduks] (die Truppen von Idamaraz, Emutbal, Uruk und Isin mit Waffen geschlagen).`(=10. Regierungsjahr Samsu-ilunas)

Siegelabrollung:

1 Mannum-mēšu-liș[șur],

2 Sohn von Awilìj[a].

3 Munawwirum,

4 Sohn von Nuska-a[mah]. 


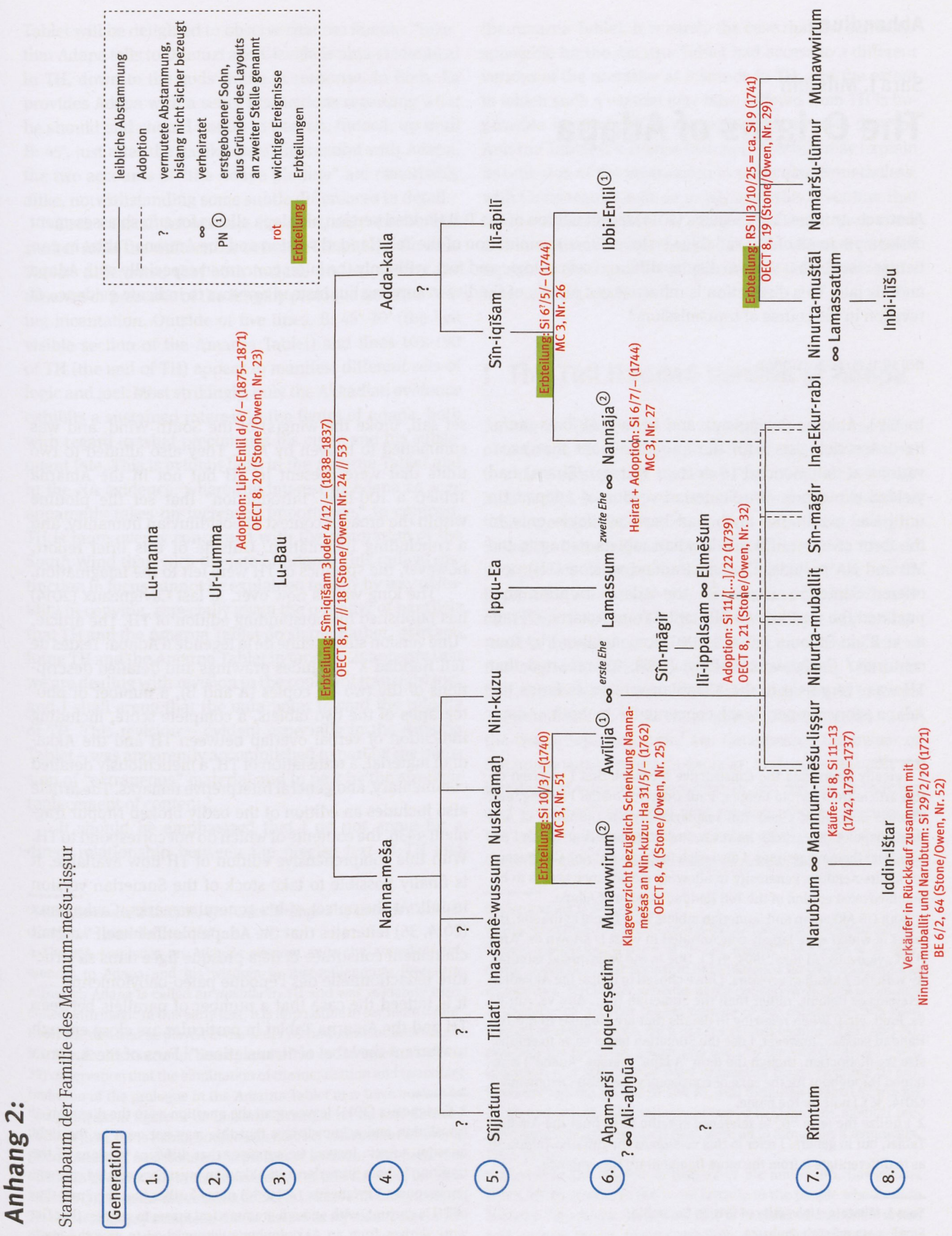

